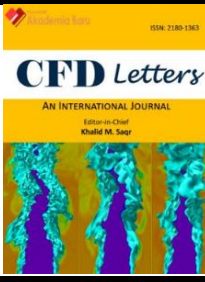

\title{
Numerical Investigation on the Pressure Drag of Some Low- Speed Airfoils for UAV Application
}

\author{
Aideal Czar Zohary ${ }^{1}$, Waqar Asrar ${ }^{1,}{ }^{*}$, Mohammed Aldheeb $^{1}$ \\ Department of Mechanical Engineering, Kuliyyah of Engineering, International Islamic University Malaysia, 53100 Kuala Lumpur, Malaysia
}

\section{\begin{tabular}{l} 
ARTICLE INFO ABSTRACT \\
\hline
\end{tabular}}

\section{Article history:}

Received 24 December 2020

Received in revised form 15 February 2021

Accepted 23 February 2021

Available online 27 February 2021

\section{Keywords:}

Low-speed UAV; SST Model;

Intermittency Transition Model; URANS
Progressive advancements in small to medium-sized fixed-wing UAVs call for prototype designing to be fast, accurate, and economical. This requires the numerical assessment of airfoil performance to be based on high fidelity replication of wind tunnel data. Furthermore, integration of drag reduction techniques is attractive as improvements in endurance, payload capacity and reduction in carbon footprints can be attained. Since a variety of suitable airfoil geometries are currently available for this application, selecting a fitting candidate can be difficult therefore risking potential gain in efficiency. The aim of this paper is to assist in resolving this issue by investigating the variation in pressure drag and its distribution with respect to the type of airfoil geometry, angle of attack, and the contribution of pressure towards the total drag at low Reynolds numbers. The airfoils selected in this study comprises of the NACA 4415, FX 61-184, E420 and S1223 which are preferred for subsonic UAV applications in addition to having the NACA 0012 serving as a standard profile. Performance of the S1223 airfoil was examined at a chord-based Reynolds number of $0.3 \times 10^{6}$ with the remaining airfoils at $1.0 \times 10^{6}$ for a range of angle of attack of around $0-10^{\circ}$. The unsteady 3-equation Intermittency SST model from ANSYS FLUENT 2020 was utilized with gradual reduction of timestep from $0.001 \mathrm{~s}, 0.0005 \mathrm{~s}, 0.00025 \mathrm{~s}$ and $0.0001 \mathrm{~s}$. Experimental lift and drag validation across the airfoils generally suggest that the transitional model regularly outperforms XFOIL. Among the selection, concave airfoils such as the E420 and S1223 excel in delivering high lift at the expense of an increase in drag. Evaluation of the $\mathrm{l} / \mathrm{d}$ ratio alone may underestimate their potential. Hence, further studies should focus on the implementation of drag reduction techniques on concave airfoils to enhance their performance. At the maximum tested angle of attack, the E420 reaches a c, value of 2.09 and S1223 at 1.98 while the FX 61-184 only at 1.57 and NACA 4415 at 1.36 .

\section{Introduction}

The name UAV covers all vehicles, which are flying in the air with no person on-board with the capability of controlling the aircraft as described by Eisenbeiss [1]. The focus of application has been

\footnotetext{
* Corresponding author.

E-mail address: waqar@iium.edu.my (Waqar Asrar)
} 
directed mostly towards military and commercial surveillance, search and rescue as well as research purposes according to Shakhatreh et al., [2]. Currently, great attention is given to the development of UAVs for improved performance, manoeuvrability, navigation, and stability. The cost of operation of a UAV can be reduced with airfoil optimization and improvements in the vehicle's aerodynamic efficiency. Considerations when selecting an airfoil for a UAV include a high maximum lift coefficient $\left(c_{\max }\right)$, high lift-to-drag ratio $\left(c_{1} / c_{d}\right)$, high endurance factor $\left(c_{1} 3 / 2 / c_{d}\right)$, and mild stall characteristics as suggested by several authors [3-5]. Over the years, a wealth of airfoils have been uniquely tailored to deliver optimal performance for their intended mission. The NACA 4415 for instance not only serves the American unmanned aerial vehicle such as the AAI RQ-2 Pioneer but also has been seen included in the structural analysis investigation for the implementation of tubercles by Adi Azriff Basri et al., [6]. The Akaflieg Darmstadt D-38,39 and 39b German sailplanes on the other hand adopted the classical FX 61-184 Wortmann series as referred to in Althaus et al., [7] which features a concavetype of pressure recovery to reduce the severity of increased drag with increasing angle of attack. Some years later, improved high lift airfoils were designed based on this concept and highlights include the E420 airfoil by Eppler [8] which is recommended for operations that exceed a Reynolds number of one million and the S1223 preferred for smaller UAVs by Selig et al., [5]. All in all, since small to medium-sized UAVs typically operate between a Reynolds number of $3 \times 10^{4}$ to $2 \times 10^{6}$ as shown by several researches $[3,5,9]$, these airfoils have great potential to be further improved with drag reduction techniques thus close investigation on their pressure drag distribution is essential.

Identifying and evaluating individual components of drag is difficult through experimental procedure, hence resorting to computational fluid dynamics (CFD) is the way to go. Reasonable aerodynamic characterizations which include transitional flow have been proven in the literature to be conveniently accessed through numerical simulations. Validation of the Transition SST model was conducted by Langtry et al., [10] using the Aerospatial A airfoil at an angle of attack of $13.1^{\circ}$ and Reynolds number of $2.07 \times 10^{6}$. The resulting data agreed well with the experimental data which successfully predicted separation-induced transition. In another study, the Transition SST model was thoroughly examined in predicting the NLF(1)-0416 and the $\$ 809$ airfoil aerodynamics at $2 \times 10^{6} \leq \operatorname{Re}$ $\leq 4 \times 10^{6}$ and $-10^{\circ} \leq \alpha \leq 15^{\circ}$ by Khayatzadeh et al., [11]. The model was able to be compared very well against wind tunnel data and the key contribution of their work includes recalibrating the intermittency correlation which improved accuracy but limited to the higher end of the Reynolds number tested. The use of this transitional model was also extended to the development of a 3D Horizontal Axis Wind Turbine model by Lanzafame et al., [12]. The empirical correlations of the Transitional SST turbulence model were modified through a significant number of numerical 2D airfoil tests and managed to obtain errors of less than $6 \%$ for all simulations when compared with experimental data. In summary, the use of the transitional model has shown to be consistent in delivering sufficient prediction of the overall performance of airfoils, hence deserve to be noted as a reliable tool of assessment.

The literature survey conducted in this work has revealed that the NACA 4415, FX 61-184, E420 and S1223 airfoils are promising candidates to be included in the design of small to medium-sized UAVs. Within the knowledge of the authors, no faithful validation and close inspection on the breakdown of drag have been performed for these airfoils at the corresponding Reynolds number. Acquiring sound understanding in the association of airfoil geometry and the production of drag as well as its decomposition will be essential for designers to effectively implement suitable drag reduction techniques. The current work will therefore concentrate on presenting the relationship between airfoil geometry and its corresponding effect on pressure drag. Numerical investigations will be performed at a chord-based Reynolds number of $0.3 \times 10^{6}$ for the $\$ 1223$ airfoil and $1.0 \times 10^{6}$ for the remaining airfoils. The range of angle of attack will be from around 0 to 10 degrees. Results from 
the simulation will be validated against XFOIL and available experimental wind tunnel data where possible. Additionally. the $c_{f}$ and $c_{p}$ distribution will also be included to allow characteristic differences between XFOIL and the FLUENT simulation to be pointed out where necessary.

\section{Numerical Methods}

\subsection{Numerical Algorithm}

Since the importance of transition properties have been highlighted, this paper will adopt the extended version of the $k-\omega$ SST model which features an additional transport equation for intermittency [13]. This model drops the transport equation for the transition momentum-thickness Reynolds number, $\operatorname{Re}_{\theta T}$ which essentially makes it a reduced form of the four-equation Transition SST by Menter et al., [14]. To appreciate the selection of the Intermittency SST model used in this study, the underpinning relevance and conceptual understanding of the governing equations will be described in brief since further information can be found in the previous studies [13, 15].

$$
\begin{aligned}
& \frac{\partial}{\partial t}(\rho \gamma)+\nabla \cdot\left(\rho U_{j} \gamma\right)=\nabla \cdot\left(\left(\mu+\frac{\mu_{t}}{\sigma_{\gamma}}\right) \nabla \gamma\right)+P_{\gamma}-E_{\gamma} \\
& P_{\gamma}=F_{\text {onset }} F_{\text {length }} S \rho \gamma(1-\gamma) \\
& F_{\text {onset }}=f\left(F_{\text {onset } 1}, F_{\text {onset } 2,}, F_{\text {onset } 3}\right) \\
& F_{\text {onset } 1}=\frac{\operatorname{Re}_{v}}{2.2} \operatorname{Re}_{\theta c}
\end{aligned}
$$

The intermittency transport equation as in Eq. (1) will be discussed first. The relaminarization term $E_{\gamma}$ will not be considered since this phenomenon is not related to this study. On the other hand, the production term of intermittency, $P_{\gamma}$ is defined in Eq. (2) where $S$ is the strain rate magnitude and the influence of intermittency is dependent on the $F_{\text {onset }}$ and $F_{\text {length. Based on Eq. (4), when the local }}$ vorticity Reynolds number, $\mathrm{Re}_{v}$ exceeds the local transition onset criteria, the production term will be switched on and kinetic energy will be discharged into the boundary layer. In addition to that, the strength of $P_{\gamma}$ is controlled by the transition length function, Flength whereby stronger production of intermittency will cause the transition length to be shorter and vice versa. The maximum value of intermittency is 1 as constrained by the last term in Eq. (2). As the intermittency saturates and reaches a maximum, the flow becomes turbulent and the original $k-\omega$ SST model will be used. Further information that describes $F_{\text {onset }}$ as seen in Eq. (3) can be referred to in the previous study [13].

As mentioned, the main difference between the Intermittency SST model and the Transition SST model lies in the presence of the transport equation for the transition momentum thickness Reynolds number. Before going further, it must be noted that both formulations use the momentum thickness Reynolds number, $\operatorname{Re}_{\theta}$ as a representation of length from the leading edge to allow general geometries to be used. Apart from that, since instabilities must first grow before transition can occur, this gives us some sense that the intermittency has to grow within the laminar boundary layer. The critical momentum thickness is defined as the location where intermittency starts to grow and is depicted in Figure 1. In the Intermittency SST model, the formulation of the critical momentum thickness Reynolds number is given in Eq. (5) where $T u_{\llcorner}$and $\lambda_{\theta\llcorner}$ are locally defined variables that approximate the freestream turbulence intensity and pressure gradient parameter respectively. $F_{P G}$ 
accounts for the influence of the freestream pressure gradient on transition as formulated in Eq. (8). Note that $\mathrm{C}_{\mathrm{TU1}}, \mathrm{C}_{\mathrm{TU} 2}, \mathrm{C}_{\mathrm{TU} 3}, \mathrm{C}_{\mathrm{PG} 1}, \mathrm{C}_{\mathrm{PG} 2}$, and $\mathrm{C}_{\mathrm{PG} 3}$ are constants and have been calibrated against a wide range of generic as well as turbomachinery aeronautical cases.

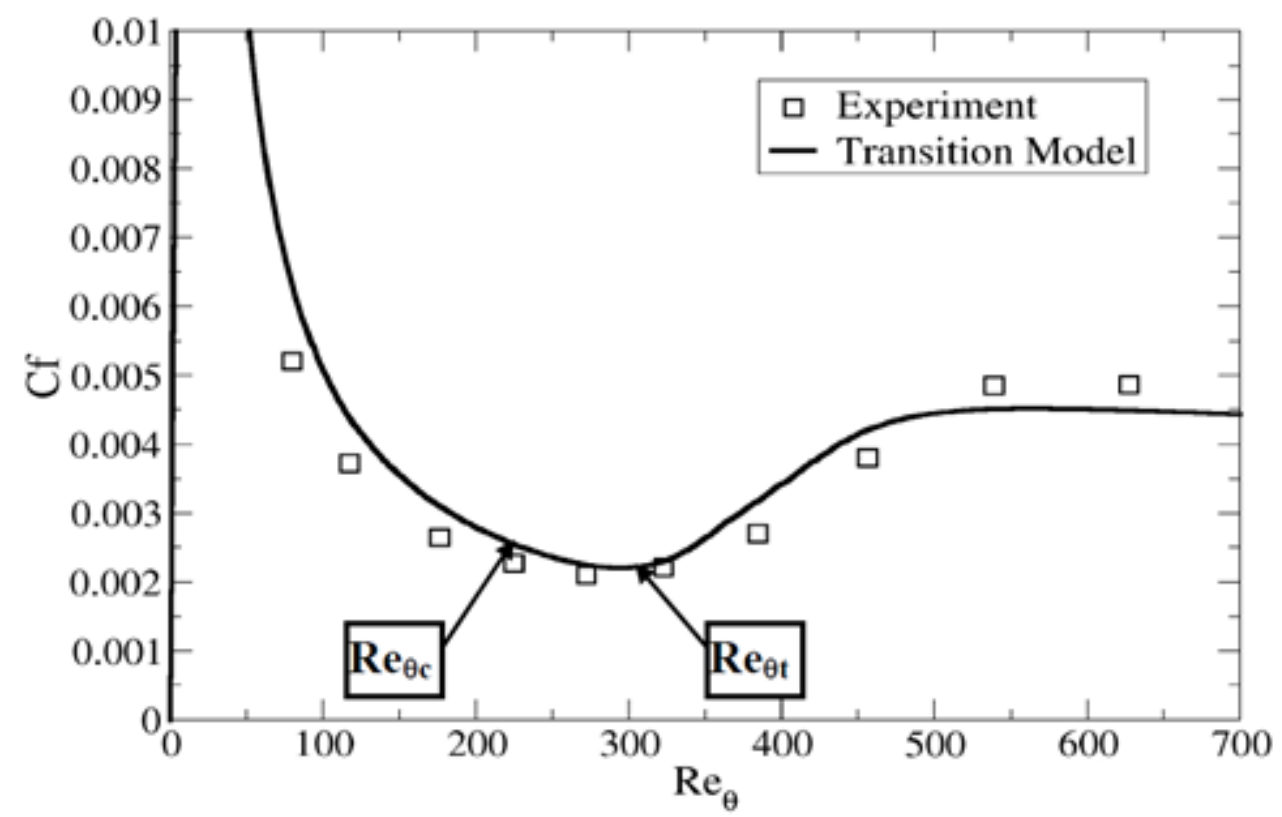

Fig. 1. Difference between critical momentum thickness Reynolds number, $\left(\operatorname{Re}_{\theta c}\right)$ where intermittency starts to grow and transition momentum thickness Reynolds number, $\left(\mathrm{Re}_{\theta \mathrm{T}}\right)$ where skin friction starts to increase [15]

$R e_{\theta c}\left(T u_{L}, \lambda_{\theta L}\right)=C_{T U 1}+C_{T U 2} \exp \left[-C_{T U 3} T u_{L} F_{P G}\left(\lambda_{\theta L}\right)\right]$

$T u_{L}=\min \left(100 \frac{\sqrt{2 k / 3}}{\omega d_{w}}, 100\right)$

$\lambda_{\theta L}=-0.1111 \frac{d V}{d y} \frac{d_{w^{2}}}{v}+0.1875$

$F_{P G}\left(\lambda_{\theta}\right)=\left\{\begin{array}{cc}\min \left(1+C_{P G 1} \lambda_{\theta}, C_{P G 1}^{\lim }\right), & \lambda_{\theta} \geq 0 \\ \min \left(1+C_{P G 2} \lambda_{\theta}+C_{P G 3} \min \left[\lambda_{\theta}+1,0\right], C_{P G 2}^{\lim }\right), & \lambda_{\theta}<0\end{array}\right.$

In brief, the Transition SST model uses experimental correlation to compute the $\operatorname{Re}_{\Theta т}$ in the freestream, and diffuses this information into the boundary layer. Then, this will be used to calculate the transition length, Flength and critical momentum thickness Reynolds number, $\operatorname{Re}_{\theta c}$. Consequently, the intermittency equation which controls the state of the boundary layer can be solved.

Conversely, the Intermittency SST model relies on the approximation of the freestream values for turbulence intensity and pressure gradient using local quantities to obtain $\operatorname{Re}_{\theta c}$ and therefore solving for intermittency. However, information was not given on how the Intermittency SST model formulates Flength. Possibly, since the association between $\operatorname{Re}_{\theta c}$ and $\operatorname{Re}_{\theta \top}$ has been established in the Transition SST, a similar linkage was devised and being employed. As the Intermittency SST model has only 3 transport equations to be solved and at the same time was developed using the Transition SST model, its economical requirement and well-grounded formulations make it an excellent choice for fast and reasonably accurate computations. 


\subsection{Grid Information}

Preparation of the geometry was done using Solidworks 2019 student version and proceeded with structured meshing using ICEM from the ANSYS 2020 R2 software package. Airfoils in this study have a sharp trailing edge and coordinates were extracted from the UIUC Airfoil Coordinates Database. The designated value for $\mathrm{y}^{+}$was chosen to be 1 based on the flat plate assumptions and post-processing plot for corresponding values indeed shows that the values are less than 1 around the airfoil as required by Menter et al., [14]. The C-type topology was applied with the radius of the semi-circle boundary being 30 chord lengths and the wake region extending 60 chord lengths downstream as seen in Figure 2. Also, boundary conditions comprise of the velocity inlet as denoted in blue and pressure outlet in red. The curvilinear mesh includes guide curves to allow an effective blocking strategy. The standard design procedure is having the first "outer shell" to be $1 / 30$ chord length away from the airfoil and normal lines will be drawn to later assist the mesh to be almost always orthogonal to the airfoil especially in areas of high curvature. The following number of shells will be dependent on the complexity of the geometry and serves to ease transitioning as the mesh grows away from the airfoil. The mesh criterion can be referred to in Table 1, while a closeup view of the mesh in Figure 3.

Table 1

Mesh quality of the meshes used in this study

\begin{tabular}{lll}
\hline & $\begin{array}{l}\text { 3×3 Determinant (Min) } \\
\text { Range: O(Min)-1(Max) }\end{array}$ & $\begin{array}{l}\text { Eriksson Skewness (Min) } \\
\text { Range: O(Min)-1(Max) }\end{array}$ \\
\hline NACA 0012 & 0.878 & 0.778 \\
NACA 4415 & 0.925 & 0.744 \\
FX 61-184 & 0.890 & 0.859 \\
E420 & 0.894 & 0.848 \\
S1223 & 0.839 & 0.728 \\
\hline
\end{tabular}

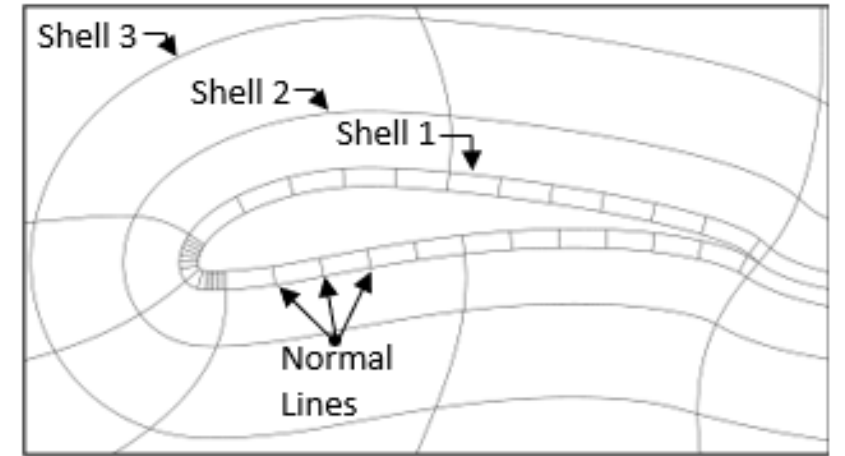

(a)

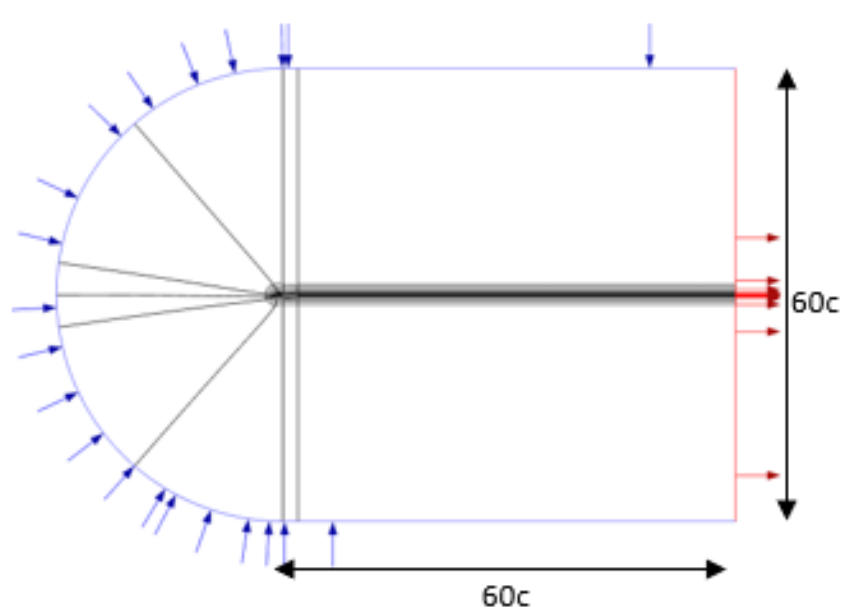

(b)

Fig. 2. S1223 grid (a) Closeup view of the airfoil with guide curves (b) Computational domain 


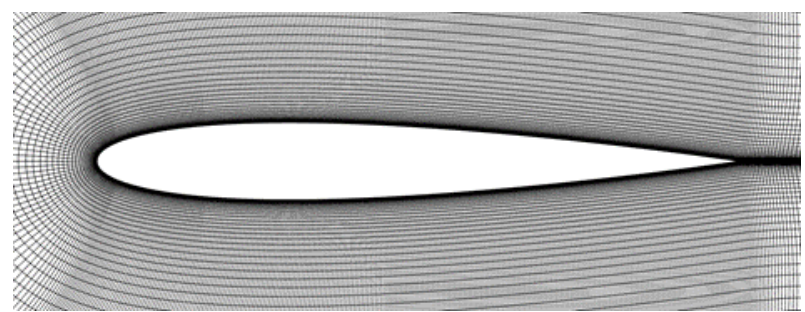

(a) NACA 0012 Moderate Mesh

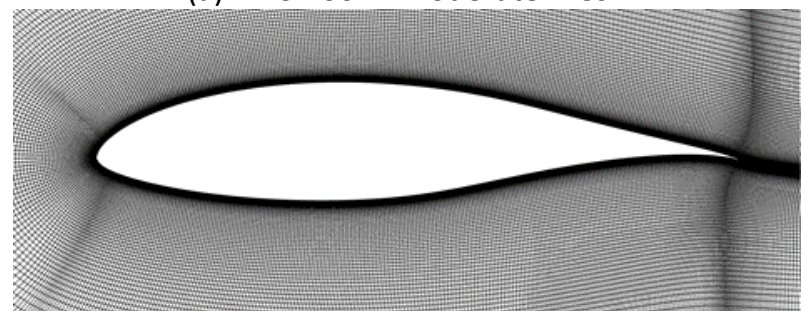

(c) FX 61-184 Moderate

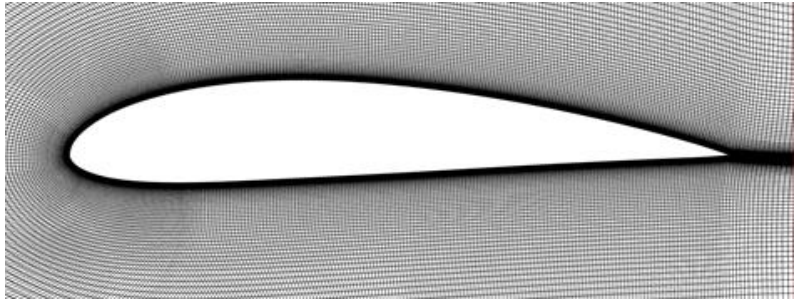

(b) NACA 4415 Moderate Mesh

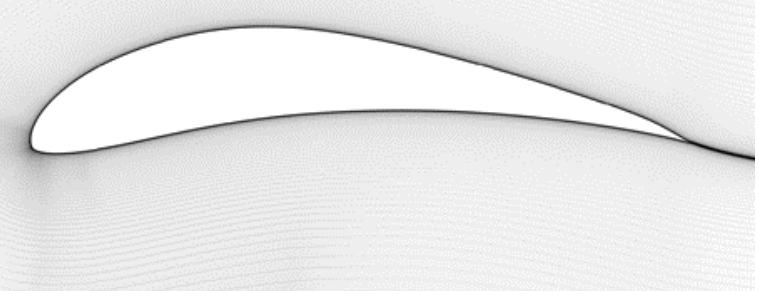

(d) E420 Fine

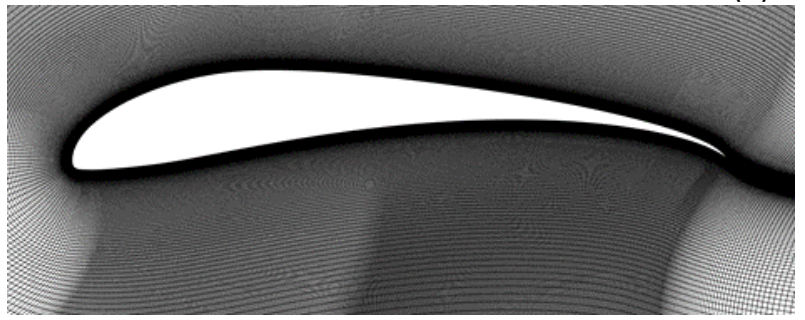

(e) S1223 Fine

Fig. 3. Close-up view on the grid

\subsection{Numerical Settings}

ANSYS 2020 R2 software package was used to carry out the transient simulations on a machine that runs on an Intel Core i5-8250U CPU @ $1.60 \mathrm{GHz}$ and has 32GB of RAM. The pressure-velocity coupling scheme was set to 'coupled' to accelerate convergence according to Keating [16] and the least-squares cell based gradient method was chosen for the spatial discretization with second-order accuracy. While the Bounded Second Order Implicit formulation provides the same accuracy as the Second Order Implicit formulation, it gives better stability and is therefore used in this simulation [13]. Turbulence intensity will be set according to available experimental data. The turbulent viscosity ratio was chosen to be 10 as was done by Langtry et al., [10]. It should be emphasized that the stability of an unsteady simulation is crucial in ensuring efficient and accurate computation. The first step is therefore to run the $k$ - $\omega$ SST model in a steady-state for 200 iterations after which the introduction of the intermittency transport equation will also be running in a steady-state for 80 iterations. At this point, the simulation was shown to be in a state where time-dependent settings should not result in strong instabilities and hence proceeded with an initial time step of 0.001 seconds with 10 inner iterations. The convergence of each timestep was decided when the change in $c_{1}$ and $c_{d}$ is small and further reduction of timestep took place in the sequence of $0.001 \mathrm{~s}, 0.0005 \mathrm{~s}, 0.00025 \mathrm{~s}$, and $0.0001 \mathrm{~s}$. Convergence in this study was decided when three conditions were met; (1) negligible change in $c_{l}$ and $c_{d}$ up to 4 decimal places, (2) properly resolved transition characteristics through monitoring the $c_{f}$ plot, and $(3)$ residual errors values of below $10^{-6}$ for all governing equations.

As mentioned, the simulation will also be compared with the XFOIL code developed by Drela [17]. The code combines a panel method and an integral boundary layer formulation for the analysis of potential and viscous flows around airfoils. The code was developed to rapidly predict the airfoil performance at low Reynolds numbers and its capabilities are well recognized [15, 18]. During validation, 200 panels were used to represent the airfoil together with a bunching parameter of 1 to 
allocate sufficient panels at the leading and trailing edge. The critical amplification ratio holds the value of 9 which represents the average wind tunnel environment.

\subsection{Mesh Independence Study}

The accuracy of the simulation depends on a number of factors which also includes the number of cells within the domain and the choice of the timestep. In this study, all five meshes have achieved mesh independence. It is well known that unsteady transitional simulation requires considerably finer mesh in comparison to the fully turbulence model running in a steady-state. Each case in this study adopted a different density of mesh to conform to their respective airfoil curvature. For example, the fine mesh used for the E420 has 286,152 cells whereas in the case of the NACA 0012 is only 143,450 . Construction of moderate mesh in this study ranges from $82,650-131,172$ cells which is already considered quite heavily dense in practice.

A systematic approach was taken in refining the mesh where the variables consist of the total number of nodes in the chordwise direction on the airfoil as well as in the viscous sublayer. The angle of attack of choice was at $9.65^{\circ}$ for the S1223 airfoil and at $9^{\circ}$ for the remaining airfoils. The reason for the choice is because, at a higher angle of attack, the degree of change in pressure gradient will be much more notable which will surely affect transition characteristics and if present, separation point. Besides that, accurate resolution of the wake will also be equally as important. Therefore, it is expected that if the resolution of the grid is sufficiently fine at a higher angle of attack, then it should also do well at lower angles. Corresponding information on the mesh independence study on the $c_{1}$ and $c_{d}$ can be referred to Table 2 and 3 respectively.

Table2

Effect of Mesh Resolution on $\mathrm{c}_{1}$

\begin{tabular}{llllll}
\hline Airfoil & NACA 0012 & NACA 4415 & FX 61-184 & E420 & S1223 \\
\hline Chordwise Nodes & 380 & 402 & 392 & 390 & 452 \\
Coarse & 0.9391 & 1.2556 & 1.3905 & 1.7263 & 1.9927 \\
\hline Chordwise Nodes & 632 & 536 & 578 & 530 & 452 \\
Moderate & 0.9460 & 1.3006 & 1.5084 & 2.0138 & 1.9963 \\
\hline Chordwise Nodes & 1642 & 986 & 846 & 1312 & 1196 \\
Fine, $\mathrm{y}^{+}=1.0$ & 0.9469 & 1.3092 & 1.5077 & 2.0239 & 1.9839 \\
\hline Chordwise Nodes & 632 & 0 & 0 & 0 & 0 \\
Fine, $\mathrm{y}^{+}=0.5$ & 0.9471 & 0 & 0 & 0 & 0 \\
\hline
\end{tabular}

Table 3

Effect of Mesh Resolution on $c_{d}$

\begin{tabular}{llllll}
\hline Airfoil & NACA 0012 & NACA 4415 & FX 61-184 & E420 & S1223 \\
\hline Chordwise Nodes & 380 & 402 & 392 & 390 & 452 \\
Coarse & 0.0141 & 0.0151 & 0.0213 & 0.0396 & 0.0317 \\
\hline Chordwise Nodes & 632 & 536 & 578 & 530 & 452 \\
Moderate & 0.0142 & 0.0128 & 0.0138 & 0.0193 & 0.0313 \\
\hline Chordwise Nodes & 1642 & 986 & 846 & 1312 & 1196 \\
Fine, $\mathrm{y}^{+}=1$ & 0.0141 & 0.0129 & 0.0137 & 0.0187 & 0.0325 \\
\hline Chordwise Nodes & 632 & 0 & 0 & 0 & 0 \\
Fine, $\mathrm{y}^{+}=0.5$ & 0.0141 & 0 & 0 & 0 & 0 \\
\hline
\end{tabular}

Unlike standard construction of mesh, the design of the curvilinear mesh in this study requires a significant number of cells in the chordwise direction to accommodate smooth transitioning. This can be seen where even in the coarse mesh, the number of nodes on the airfoils is within the range of 
$380-452$. It is clear that even with this level of resolution, it is inadequate in reaching independence. Regardless of the number of nodes in the chordwise direction, the first cell height of every mesh was set to 1 based on the assumption of a flat plate unless stated.

Even though post-processing works already revealed that the $\mathrm{y}^{+}$of all of the constructed mesh into having an actual value of close to 1 , the effect of decreasing the $y^{+}$value was still examined in the case of the NACA 0012 airfoil as a standard procedure. It can be seen that no further improvements can be attained when the moderate grid is refined by setting the $\mathrm{y}^{+}$value to 0.5 based on the flat plate assumption. This can be explained through the law of the wall where in the viscous sublayer, the relation between $\mathrm{u}^{+}$and $\mathrm{y}^{+}$is linear up to a $\mathrm{y}^{+}$value of 5 . Hence, it is futile to increase the number of nodes in the region where a linear function holds. The next step was then to increase the number of nodes in the chordwise direction which also showed minor change. This indicates that the moderate grid can properly capture sufficient detail of the pressure gradient presented on the NACA 0012. It should be expected that airfoils with higher curvature will not necessarily comply with the same resolution since the change in pressure gradient will be much greater.

Further examination was carried on the effect of refining the grid near the airfoil when the $\mathrm{y}^{+}$and spanwise nodes were kept constant. This was done on the S1223 airfoil where the number of nodes in shells 1, 2, and the immediate wake was doubled. Unsurprisingly, the results are almost the same. However, even though the change in $c_{l}$ and $c_{d}$ is small when the number of nodes on the chord has drastically increased in the fine mesh, closer inspection certainly shows an apparent difference in the skin friction coefficient plot shown in Figure 4. The fine mesh gave almost similar turbulent reattachment point onset with XFOIL rather than the coarse and moderate grid. It was mentioned in the numerical algorithm that the formulation of the critical momentum thickness Reynolds number, $\operatorname{Re}_{\theta c}$ is dependent on the pressure gradient. It can be expected that with the large increase in the number of nodes in the streamwise direction, the resolution of the pressure gradient will be improved therefore $\operatorname{Re}_{\theta c}$ will approach a stable value. Since the formulation of $F_{\text {length }}$ was not given, it is difficult to make a qualitative argument on this matter, however, it can be seen that $F_{\text {length }}$ is sensitive towards the number of nodes in the streamwise direction. This occurrence also was detected on the E420 airfoil. As a result of this, it was therefore decided that the NACA 0012, NACA 4415 , and FX 61-184 be proceeded using the moderate grid while the E420 and S1223 using the fine grid.
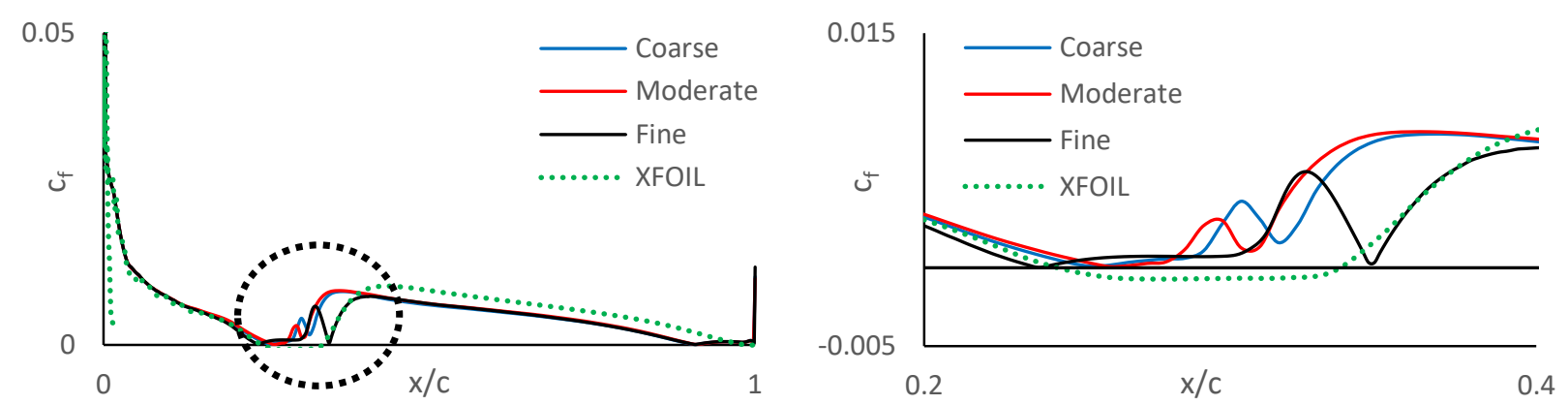

Fig. 4. Effect of grid resolution on the skin friction coefficient, $c_{f}$ on the top surface of the $S 1223$ airfoil at $\alpha: 9.65^{\circ}$ and Re: $0.3 \times 10^{6}$. (a) Global variation of $c_{f}(b)$ Variation of $c_{f}$ near transition onset

This section describes the airfoil validation of the numerical results against wind tunnel data of the NACA 0012 from Sheldahl et al., [19], NACA 4415 and FX 61-184 from Miley [20] as well as the S1223 by Selig et al., [5]. Unlike the $k-\omega$ SST, the intermittency model displayed improved prediction of the transitional region as shown in Figure 5. Results of $c_{I}$ and $c_{d}$ from the simulations in general were successful in predicting good agreement with XFOIL and wind tunnel data as can be seen in 
Figure 6. In the case of the FX 61-184, offset of the characteristics lift and drag from the experimental data can be linked to the formation of laminar separation bubble which can especially be seen at $9^{\circ}$ and $10^{\circ}$ angle of attack in Figure 7. Besides that, the difference in $c_{d}$ for the E420 airfoil stems from the presence of a laminar separation bubble using XFOIL whereas the intermittency SST predicted that the laminar boundary layer transitioned without separation which is shown in Figure 8. Clearly, the contribution of skin friction drag increases considerably. Nevertheless, since there is no experimental skin friction or pressure distribution to be compared with, no measurable accuracy can therefore be concluded on the performance of the simulation tools.

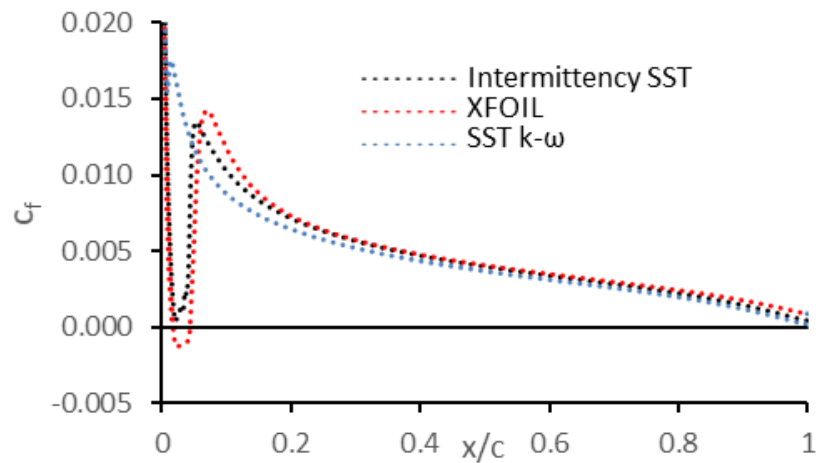

(a)

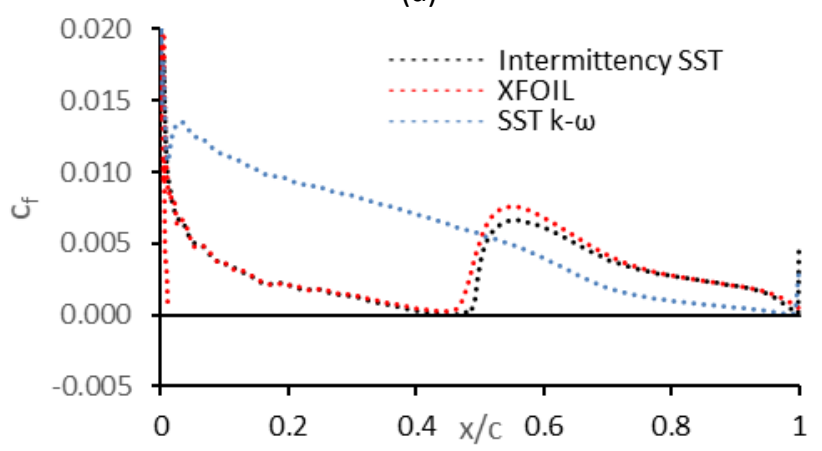

(c)

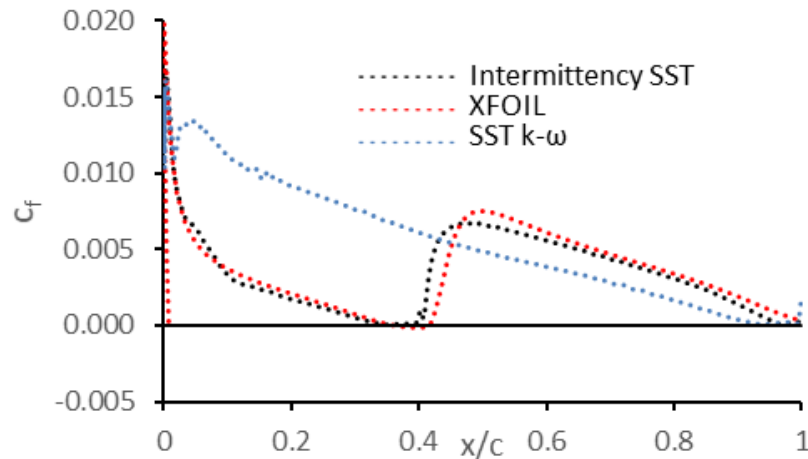

(b)

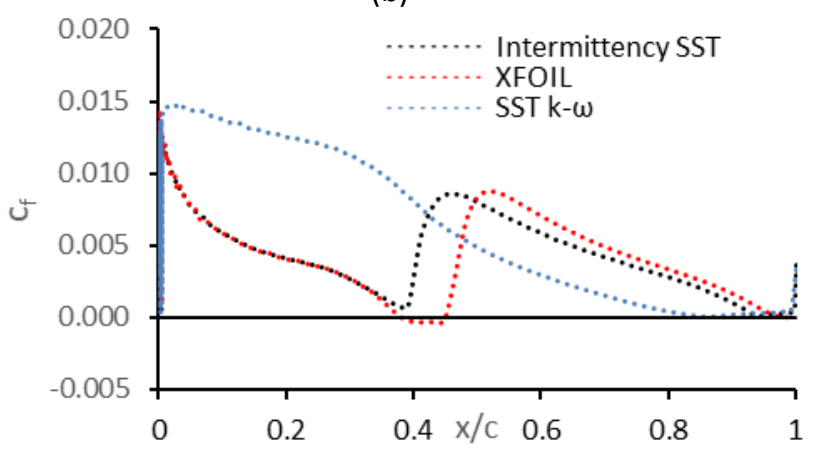

(d)

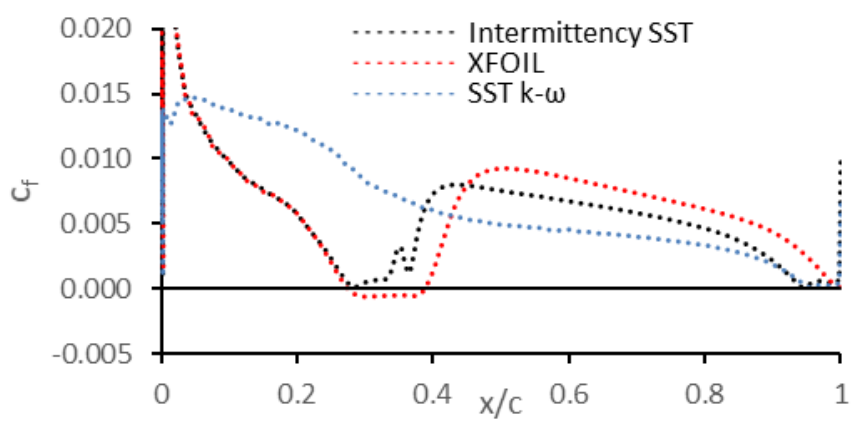

(e)

Fig. 5. Skin friction distribution on the upper surface at corresponding alpha(//d)max as a function of chord length at Re: $1.0 \times 10^{6}$ for (a) NACA 0012 (b) NACA 4415 (c) FX 61-184 (d) E420 and Re: $0.3 \times 10^{6}$ for (e) S1223 


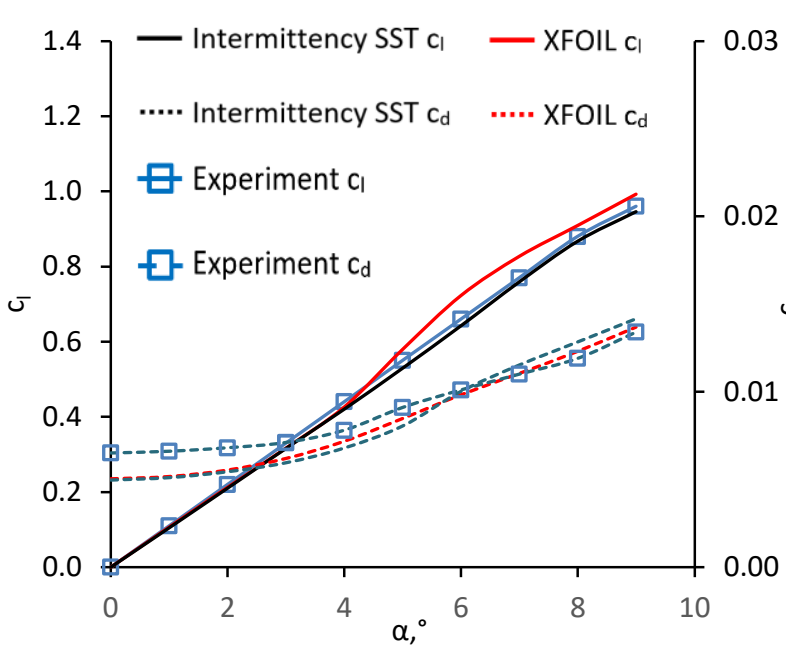

(a)

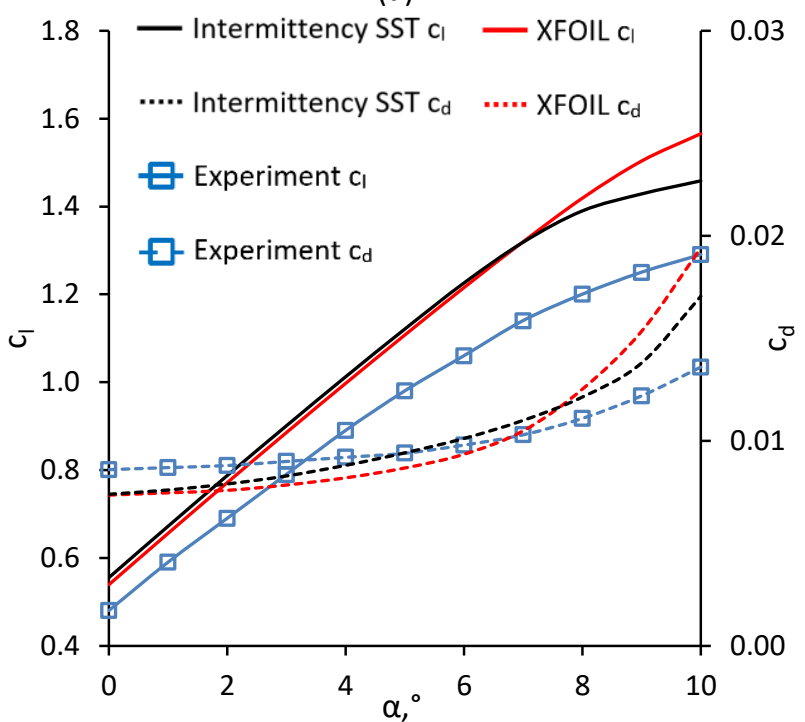

(c)

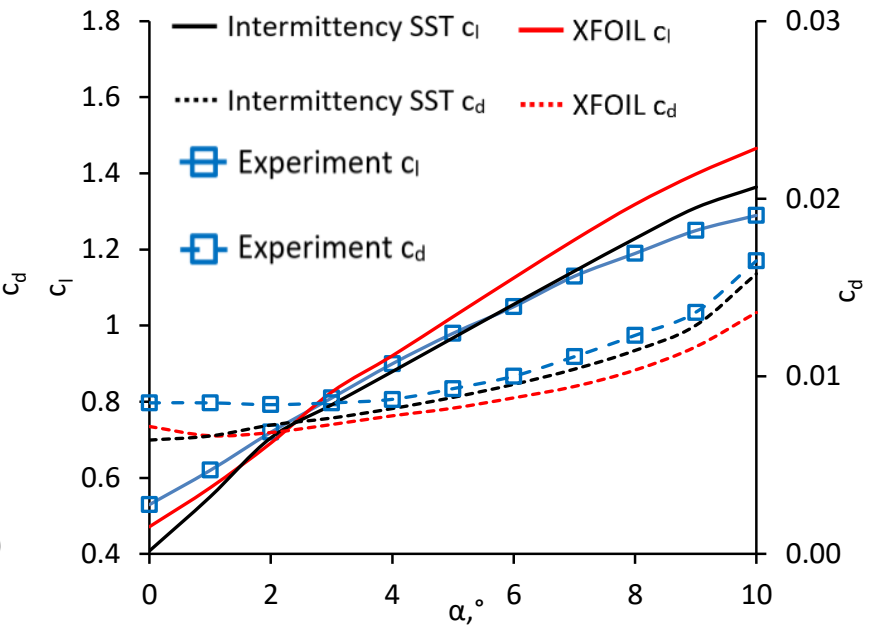

(b)

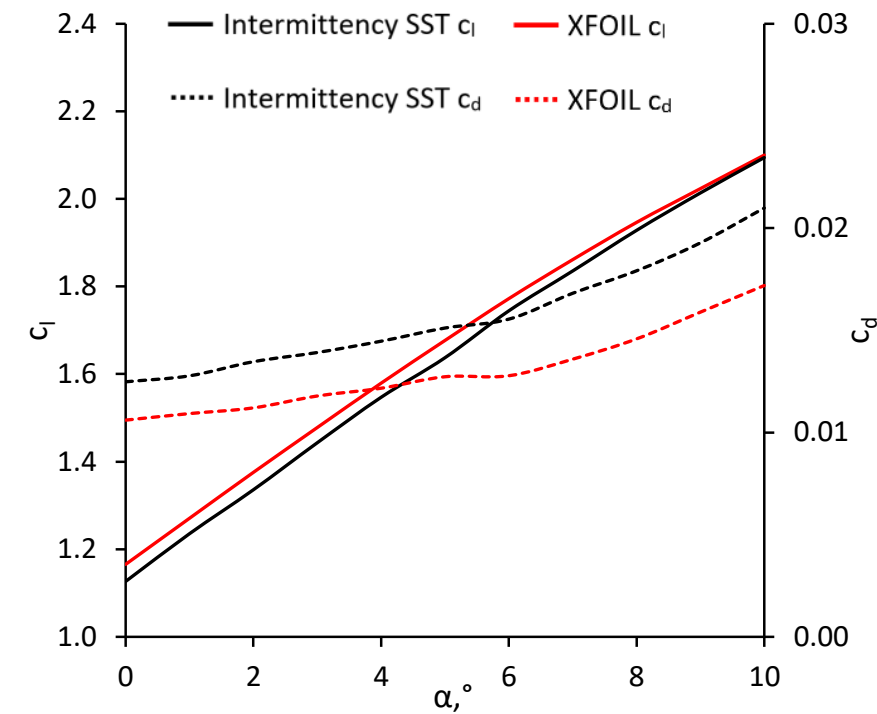

(d)

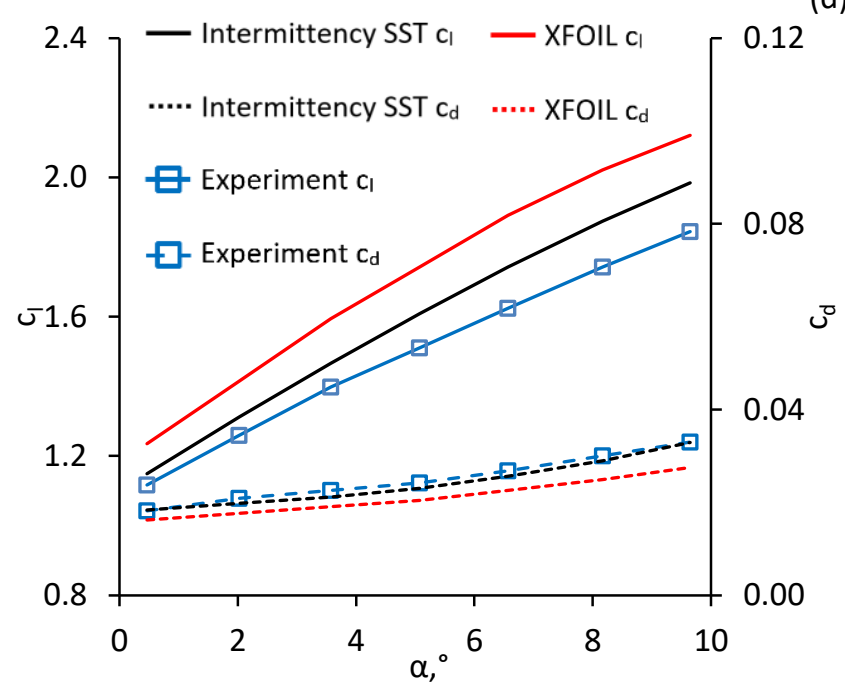

(e)

Fig. 6. Coefficient of lift and drag as a function of angle of attack at Re: $1.0 \times 10^{6}$ for (a) NACA 0012 (b) NACA 4415 (c) FX 61-184 (d) E420 and Re: $0.3 \times 10^{6}$ for (e) S1223 


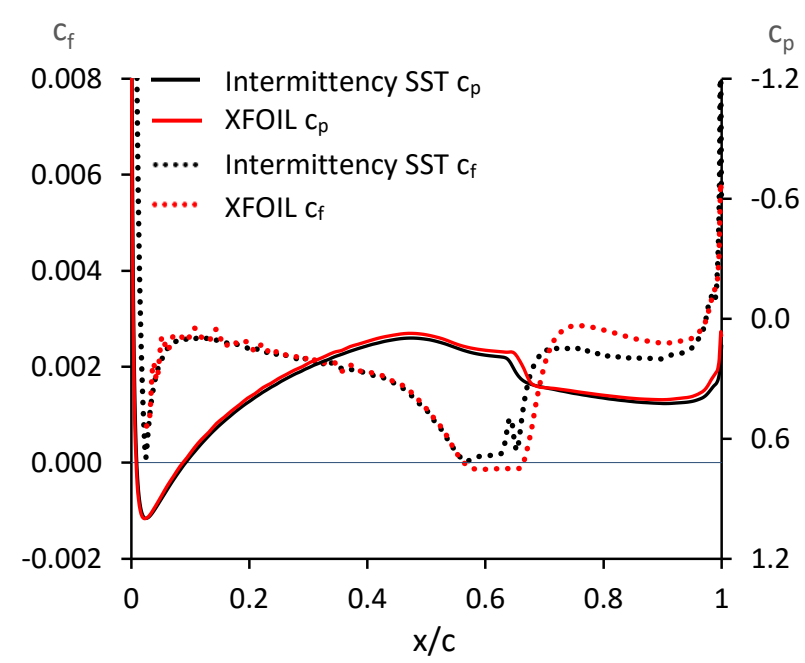

(a)

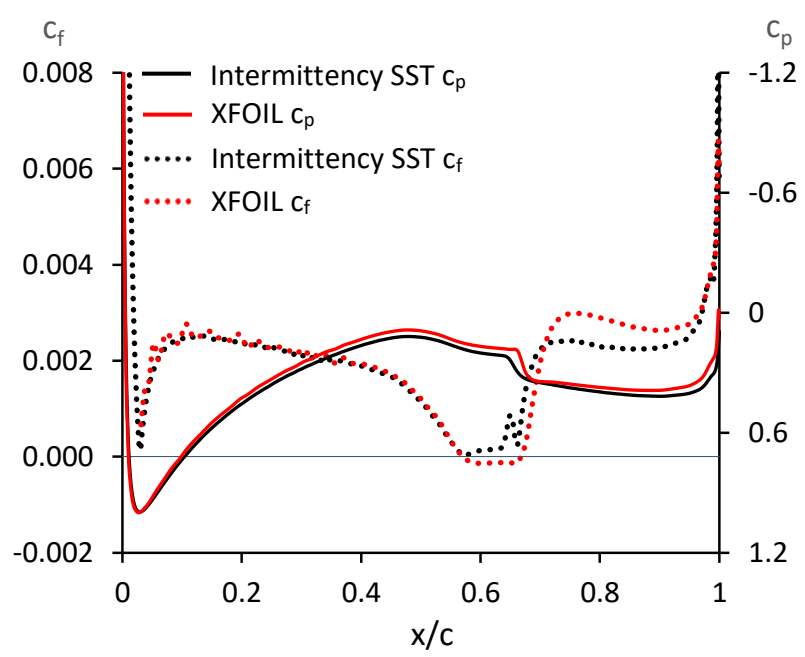

(b)

Fig. 7. Pressure and skin friction distribution over the lower surface of the FX 61-184 as a function of chordlength at Re: $1.0 \times 10^{6}$ (a) $\alpha: 9^{\circ}$ (b) $\alpha: 10^{\circ}$

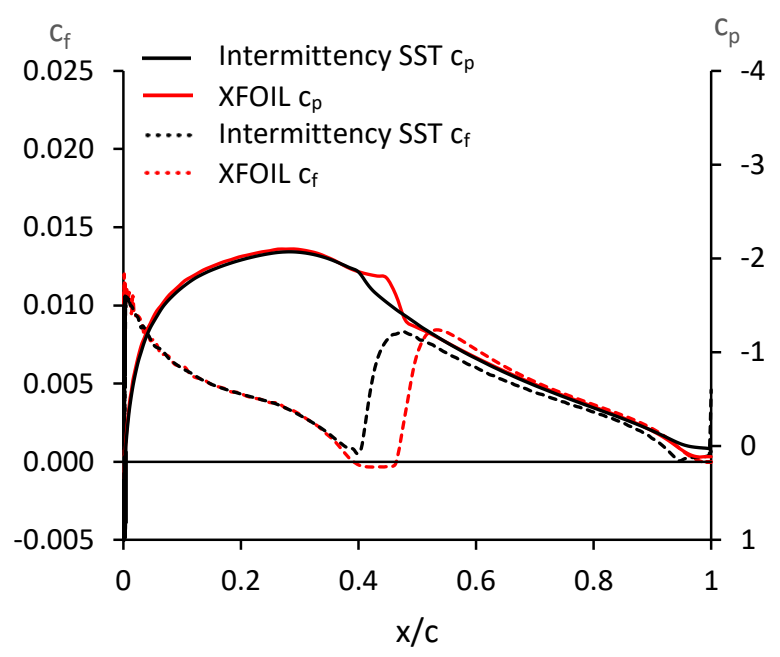

(a)

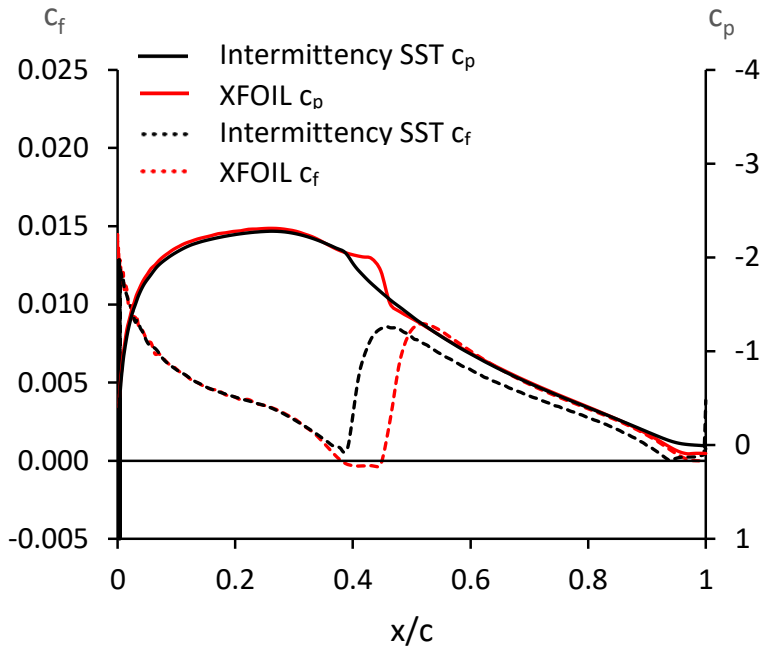

(b)

Fig. 8. Pressure and skin friction distribution over the upper surface of the $E 420$ as a function of chordlength at Re: $1.0 \times 10^{6}$ (a) $\alpha: 4^{\circ}$ (b) $\alpha: 6^{\circ}$

\section{Results and Discussions}

The first step in the analysis will be focusing on the drag composition of the airfoils. Figure 9 showcase the contribution of the components of drag in terms of percentage at the corresponding angle of attack of maximum I/d ratio in this study. It is obvious that convex trailing edge airfoil has a rather balanced influence on both components of drag which makes available skin friction drag reduction methods appear attractive in improving its performance. On the contrary, concave trailing edge airfoils display a significant contribution of pressure drag due to their generation of high lift. It should be highlighted that a high lift-to-drag ratio is not the only desirable feature of an airfoil. The airfoil maximum lift provides capability in increasing payloads, shortening the take-off and landing distances, reduced aircraft noise, and lowered stall speeds. This feature is of high value for some UAVs that operate with the airfoil near $\mathrm{C}_{1, \max }$ to achieve low-speed flight requirements for loitering, cruise, or landing. Therefore, Figure 10 (a) and (b) exhibit the trend of the change in drag composition as a function of angle of attack. All of the airfoils seem to have the same slope of change with respect 
to the angle of attack. With increasing alpha, pressure drag grows rapidly and the opposite is apparent for friction drag. The pressure drag for the $\$ 1223$ airfoil accounts for around $80 \%$ of total drag at near 10 degrees angle of attack followed by E420 at approximately $75 \%$.

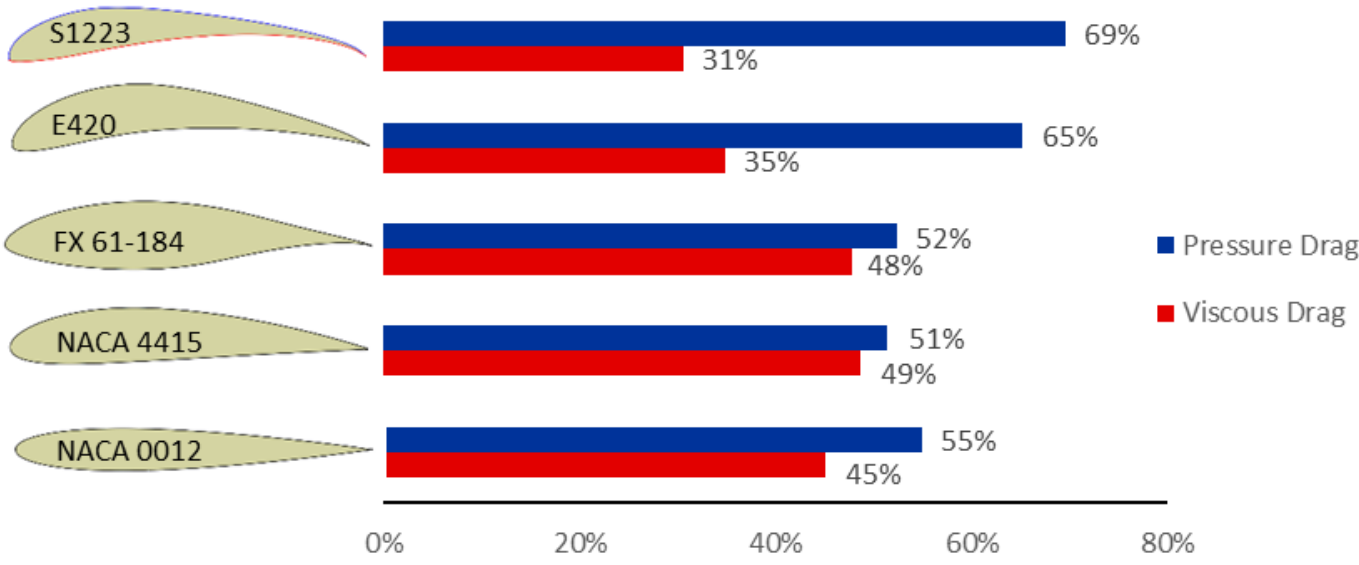

Fig. 9. Change in drag composition in terms of percentage of total drag at $\alpha_{1 / d(\max )}$ with $\mathrm{S} 1223$ at Re: $0.3 \times 10^{6}$ and all other airfoils at Re: $1.0 \times 10^{6}$

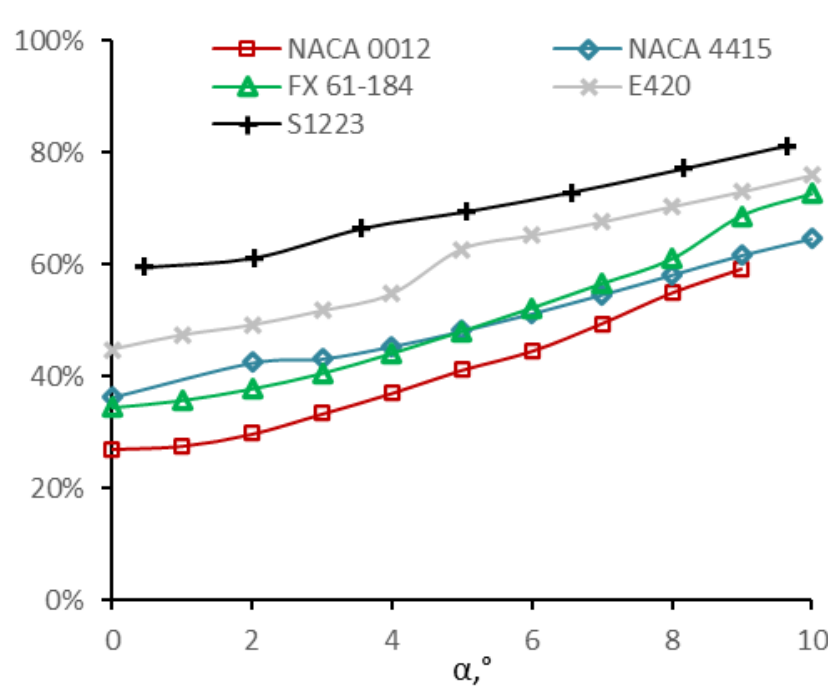

(a)

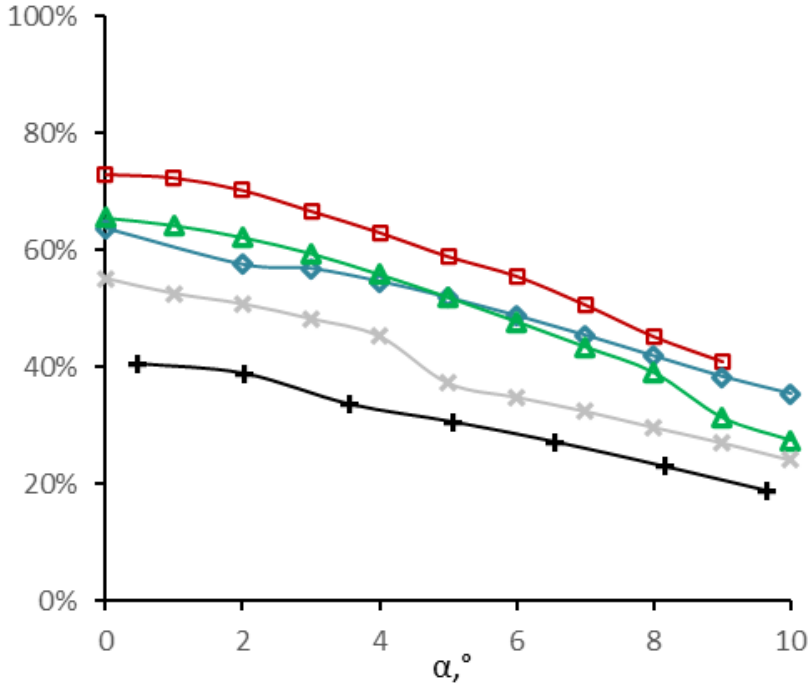

(b)

Fig. 10. Drag composition in terms of percentage of the total drag as a function of angle of attack with S1223 at Re: $0.3 \times 10^{6}$ and all other airfoils at Re: $1.0 \times 10^{6}$ (a) Pressure drag (b) Viscous drag

Evaluation of the aerodynamic performance parameters with respect to the angle of attack then can be seen in Figure 11. Despite the apparent difference in their design, the NACA 4415 and FX 61184 corresponding lift and drag variation are almost the same. On another note, it is interesting how the E420 and S1223 give very similar characteristics at their individual design Reynolds number. In addition to that, it is clear from Figure 11 (a) that the E420 and S1223 outperform the other airfoils with almost double generation of lift across the range of alpha with maximum lift were recorded to be at 2.09 and 1.98 respectively. Nonetheless, it comes with a pressure drag penalty which also results in the increase of drag to follow the same trend as shown in Figure 11 (b). In the same Figure, the $\mathrm{S} 1223$ produced the most drag primarily due to higher viscous effect at lower Reynolds number. Essentially, the qualities of the high lift airfoils are clearly not visible in Figure 11 (c), as they are shown to perform more or less the same as the other airfoils. This is simply due to the high production of 
drag that counterbalances the benefit of high lift. It is therefore attractive to improve the performance of concave airfoils which excels in delivering high lift by introducing suitable drag reduction methods.

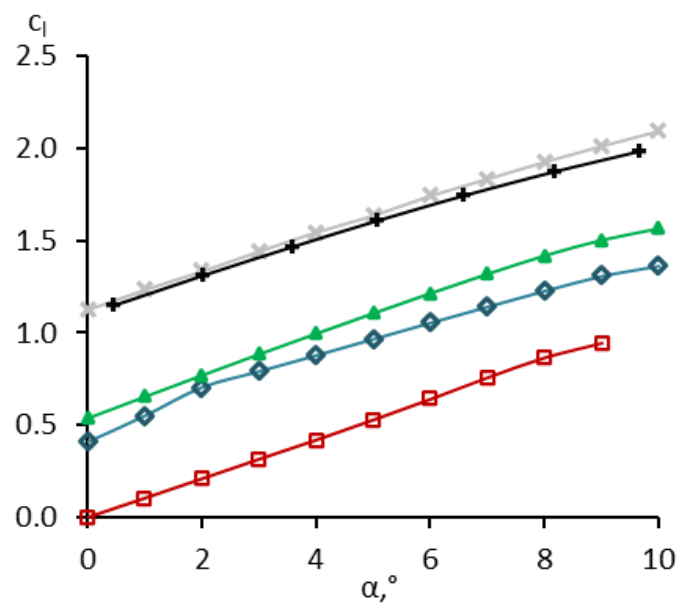

(a)

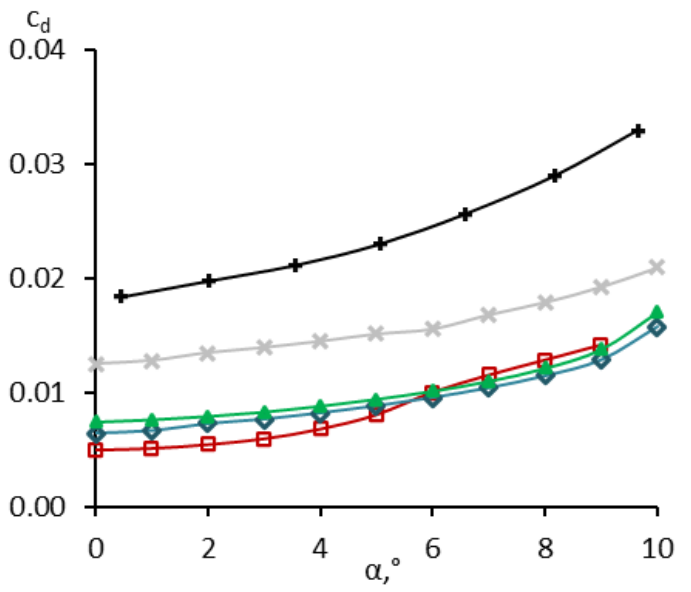

(b)

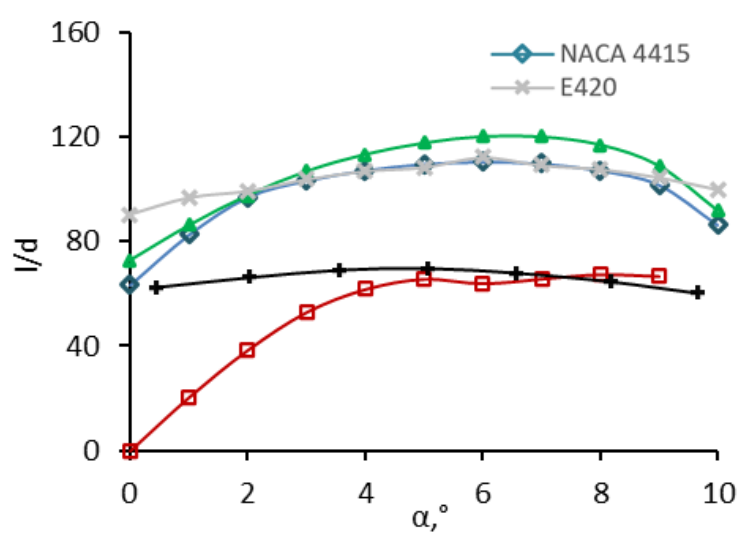

(c)

Fig. 11. Aerodynamic performance as a function of angle of attack with S1223 at Re: $0.3 \times 10^{6}$ and all other airfoils at Re: $1.0 \times 10^{6}(\mathrm{a}) \mathrm{cl}_{\mathrm{l}}(\mathrm{b}) \mathrm{c}_{\mathrm{d}}(\mathrm{c}) \mathrm{l} / \mathrm{d}$

The relationship between airfoil curvature and pressure drag will is examined next. Pressure forces have a varying degree of local contribution throughout the body. Recall that negative $c_{p}$ will cause the pressure vectors to be directed outwards from the surface and positive $c_{p}$ will cause the opposite. Apart from that, there exist normal lines from the airfoil's surface with respect to the freestream direction that will decide whether the forces contribute either towards positive drag or negative drag. From here onwards the coincident point of this line on the airfoil's surface will be referred to as the "critical point". The location where $c_{p}$ value crosses 0 results in a flip in the direction of the pressure vectors. Figure 12 interprets the $c_{p}$ distribution along the top surface of the airfoils in the form of $c_{p}$ vectors to assist visualization. The $c_{f}$ distribution is also included to demonstrate the role of the boundary layer transition in affecting the pressure forces as well. Figure 12 (e) demonstrates the previous point as the critical point on the $\mathrm{S} 1223$ at $0.46^{\circ}$ angle of attack is at 0.298 $\mathrm{x} / \mathrm{c}$ thus resulting in $70 \%$ of the pressure forces on the upper surface into having a component of drag. Apart from that, the high curvature of the airfoil as the flow reaches the trailing edge contributes to an increase in pressure drag which is less apparent in convex airfoils. This perhaps could have been an added advantage if the design of the adverse pressure gradient is capable of 
increasing the static pressure to a positive value so that the local pressure forces near the trailing edge can contribute towards negative drag instead of positive pressure drag.

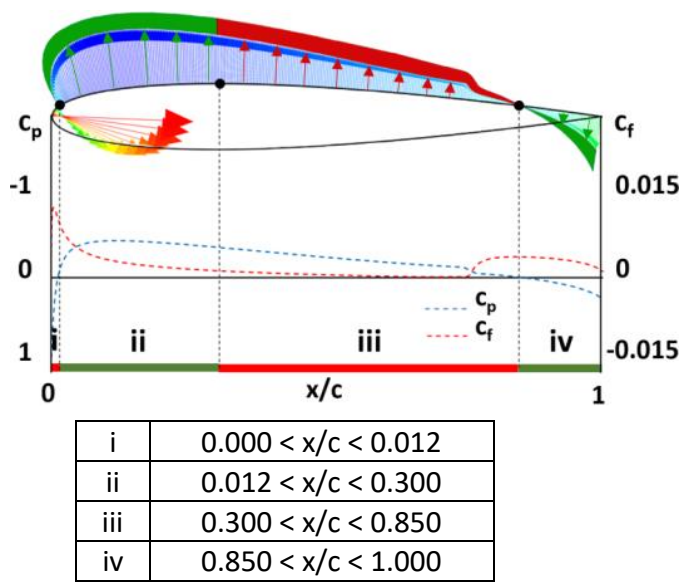

(a) NACA 0012 at $\alpha$ : $0^{\circ}$, Re: $1.0 \times 10^{6}$

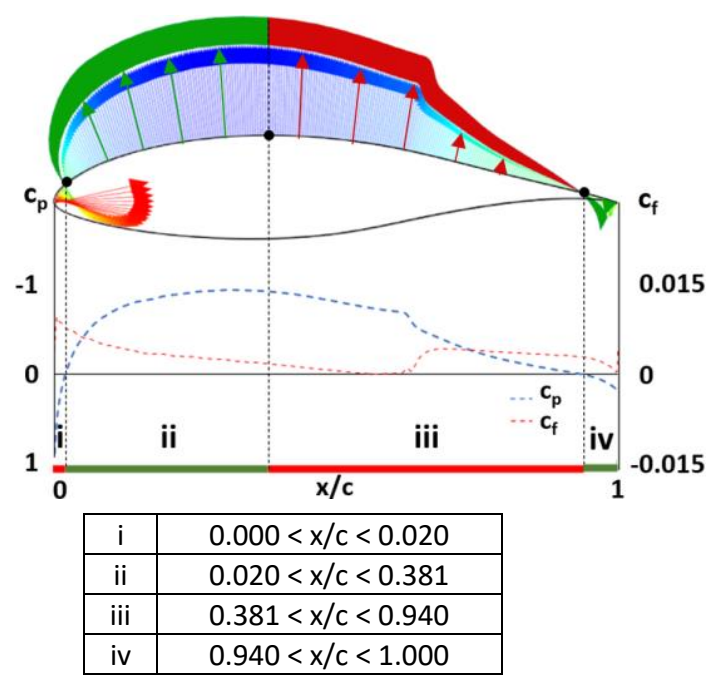

(c) FX 61-184 at $\alpha: 0^{\circ}$, Re: $1.0 \times 10^{6}$

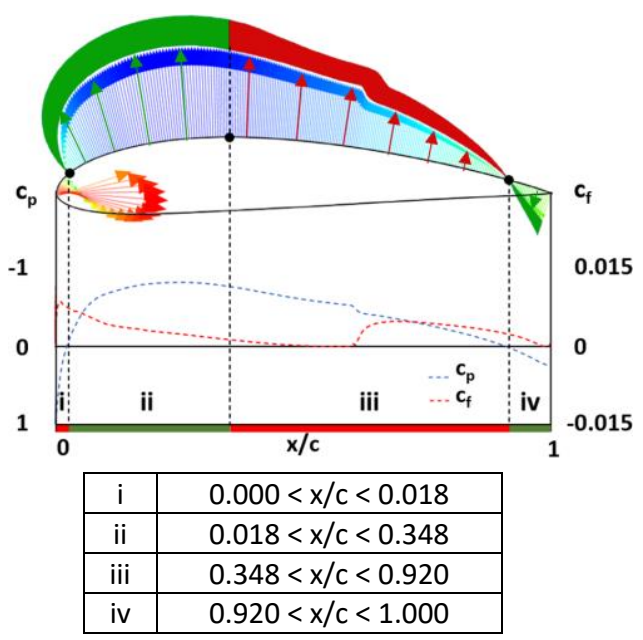

(b) NACA 4415 at $\alpha$ : $0^{\circ}, \operatorname{Re}: 1.0 \times 10^{6}$

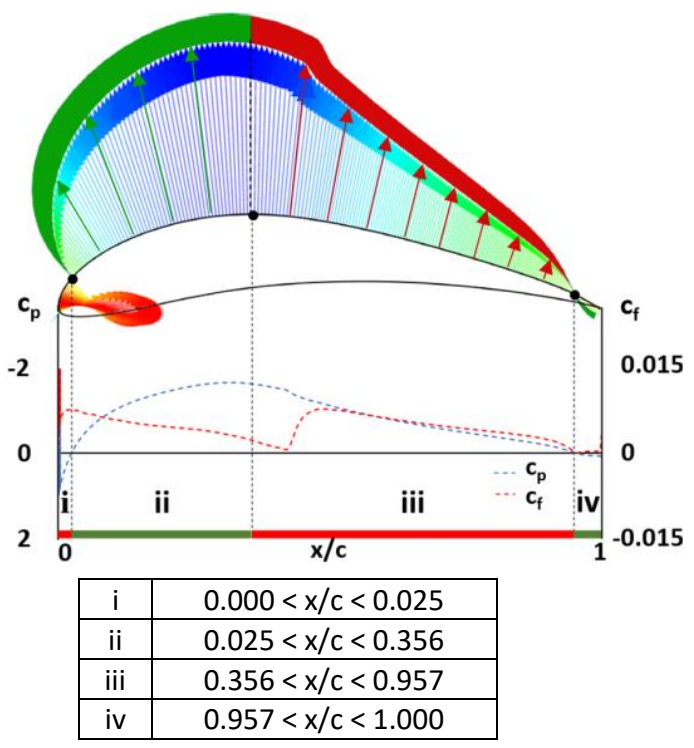

(d) E420 at $\alpha: 0^{\circ}$, Re: $1.0 \times 10^{6}$

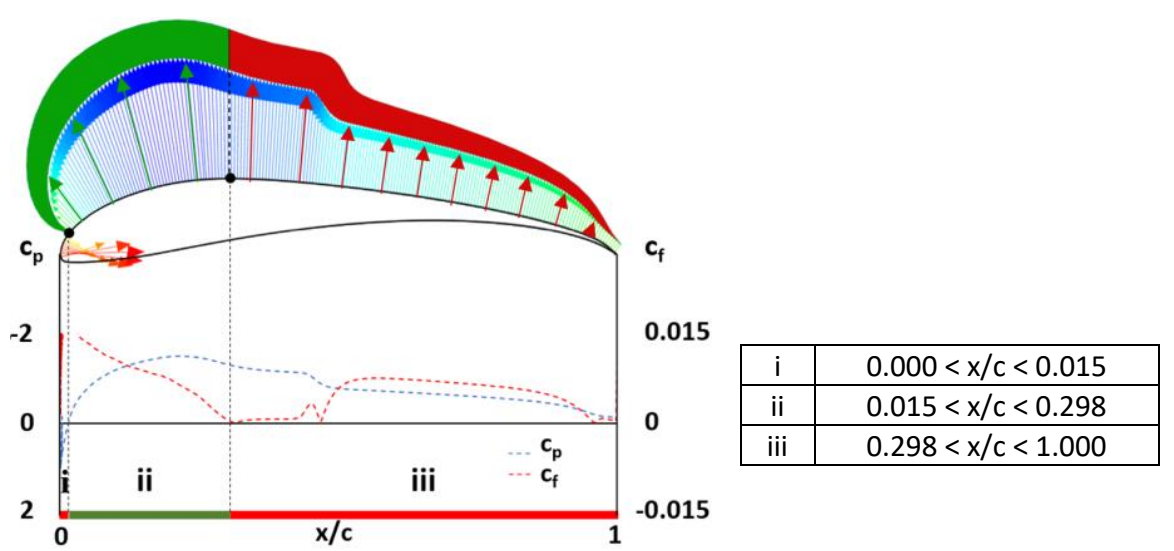

(e) S1223 at $\alpha: 0.46^{\circ}$, Re: $0.3 \times 10^{6}$

Fig. 12. Pressure vectors of the upper surface of airfoils along with $c_{p}$ and $c_{f}$ distribution as a function of chord length. Green portion represents the portion of the airfoil which contributes towards negative pressure drag and red portion represents positive pressure drag. 
As the Reynolds number increases, the flow now transitions to turbulence relatively easier as the flow instabilities grow much faster. As for the NACA 0012 in Figure 12 (a), the surface curvature is small in proportion to the boundary layer thickness, therefore the geometry has only a minor influence on the boundary layer development [21]. The NACA 4415 and FX 61-184 in Figure 12 (b) and (c) conversely take advantage of the excited instabilities by positioning the critical point slightly downstream to about $35 \%$ and $38 \%$ of the chord respectively which reduces skin friction drag via elongation of the laminar zone. As the flow eventually transitions along the adverse pressure gradient, the small increase in static pressure help to position the pressure inflection point near the trailing edge which then contributes to negative pressure drag. Consequently, region "iii" in both airfoils at 0 degrees angle of attack covers only $57 \%$ and $56 \%$ accordingly as opposed to $70 \%$ in the case of the S1223. Apart from that, since the suction peak of the FX 61-184 is greater than the NACA 4415 as to having a relatively thicker airfoil, the overall composition of pressure drag on the top surface is likely to be only by some value bigger in comparison as they very much reflect each other into having the critical point about the same location. Furthermore, when referring to Figure 12 (d) and (e), it can be said that in relation to the production of pressure drag, the E420 and S1223 airfoil have a higher composition on the top surface owing to its high lift characteristics. The sharp increase in pressure gradient before the critical point gives rise to a significantly higher suction in comparison to the other convex airfoils. The minimum value of $c_{p}$ reaches -1.66 for the E420 and -1.48 for the S1223 whereas only -0.82 and -0.93 for the NACA 4415 and FX 61-184 respectively. To add further, the relatively steep airfoil curvature gradient aft of the critical point especially for the E420 airfoil provides a substantial increase in pressure drag due to the combined effect of strong pressure suction as well as the increase in the degree of the local component of drag acting normal to the surface. The extent of the positive pressure drag region spans to be about $64 \%$ and $70 \%$ for the E420 and S1223 correspondingly which in turn causes considerable addition towards total drag. So far, it can be emphasized that the nature of pressure distribution on the upper surface of all airfoils is essentially the same. The intensity of the favourable pressure gradient depends on the vertical and streamwise position of the critical point in relation to the stagnation point. To acquire strong suction, designers should adopt a thicker airfoil relative thickness and higher camber. In connection to that, the adverse pressure gradient run too should not be relaxed rather abruptly to avoid flow separation as well as to weaken the drag component of the pressure vectors.

The next step is to study the effect of the geometry on the lower surface of airfoils in producing drag as shown in Figure 13. The NACA 0012 airfoil was not used due to symmetry therefore Figure 12 (a) can be used. With interest in the boundary layer, we can observe that concave airfoils have a rather early transition near the leading edge while convex airfoils experience the onset to be further downstream. This is anticipated as in a curved potential flow, pressure and centrifugal forces normal to the streamlines are in perfect equilibrium while in viscous flow, the latter fade out towards the wall, and the flow now becomes dynamically stratified, stable on a convex wall, and unstable on a concave wall [21]. The instability stated manifests in the form of counter-rotating vortex pairs known as Görtler vortices which provokes the boundary layer into transitioning through the coupling with the Tollmien-Schilichting waves. This also can be seen in the experimental work of Mangalam et al., [22] on a concave airfoil. For a wide range of angle of attack alpha in the case of the FX 61-184, a laminar separation bubble was seen on the lower surface near the pressure inflection point which causes higher drag in comparison to experimental data. As simulations operate in a "perfect" environment, surface irregularities and external disturbances during experimentation may result in a shorter bubble. 


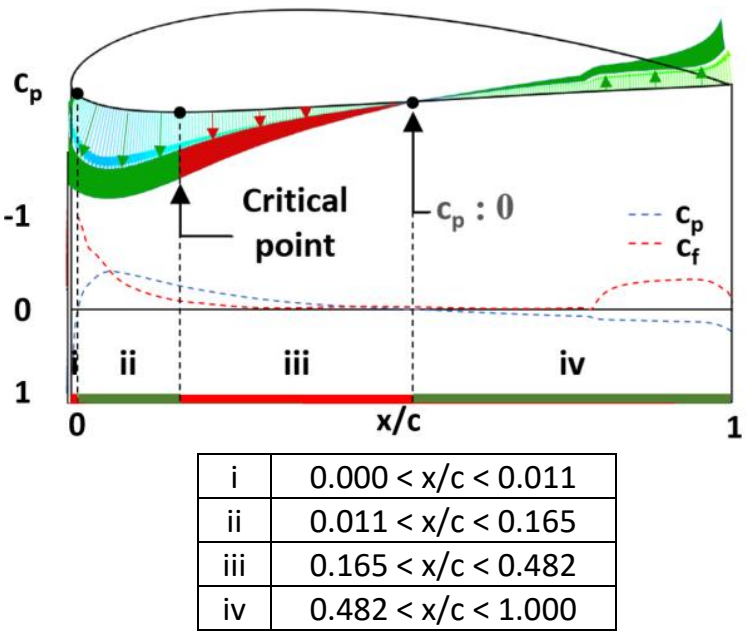

(a) NACA 4415 at $\alpha: 0^{\circ}$, Re: $1.0 \times 10^{6}$

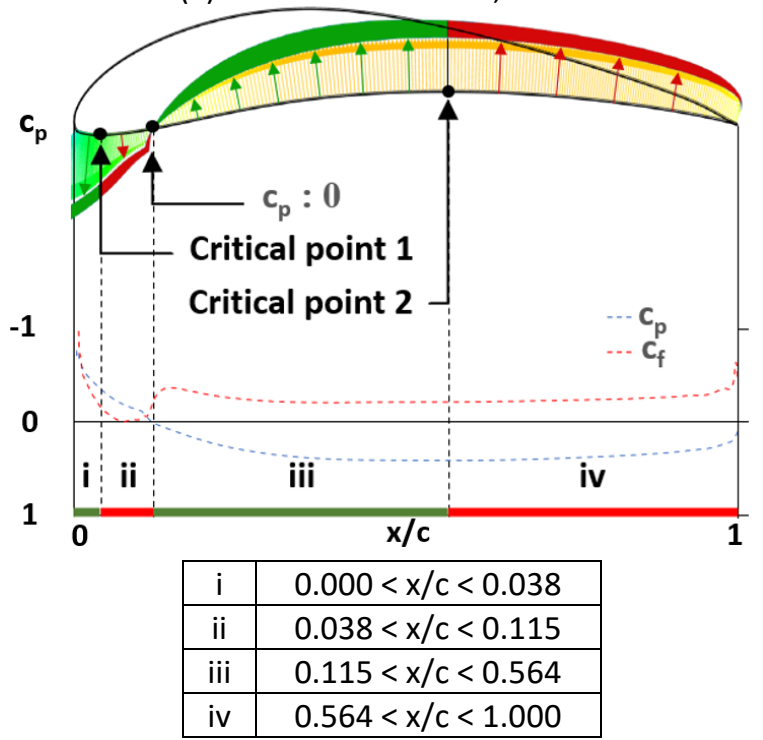

(c) E420 at $\alpha: 0^{\circ}$, Re: $1.0 \times 10^{6}$

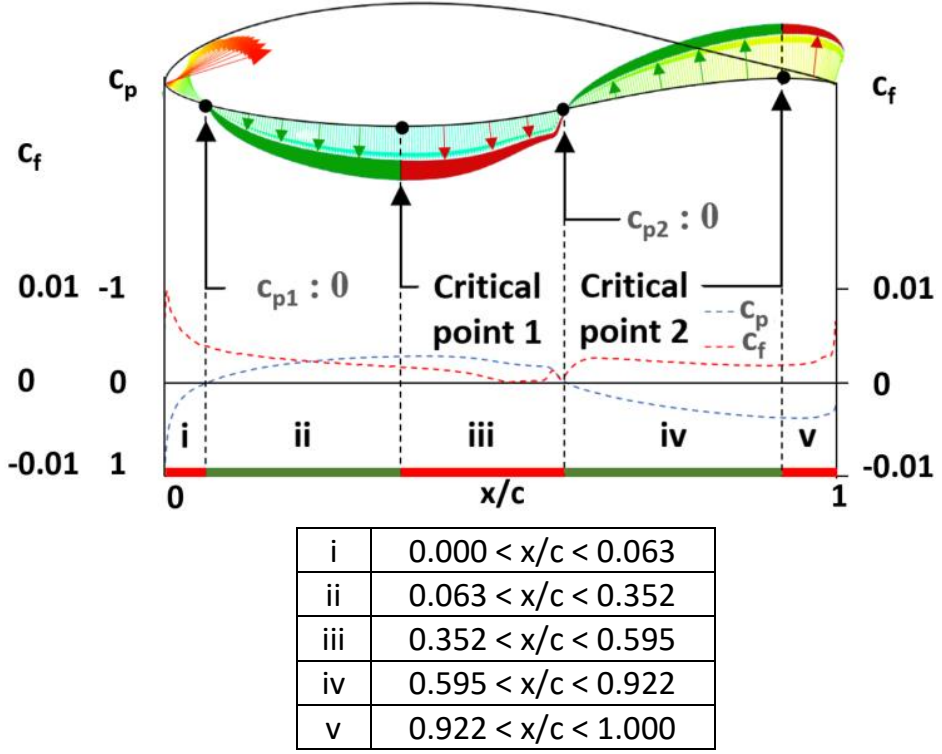

(b) FX 61-184 at $\alpha$ : $0^{\circ}$, Re: $1.0 \times 10^{6}$

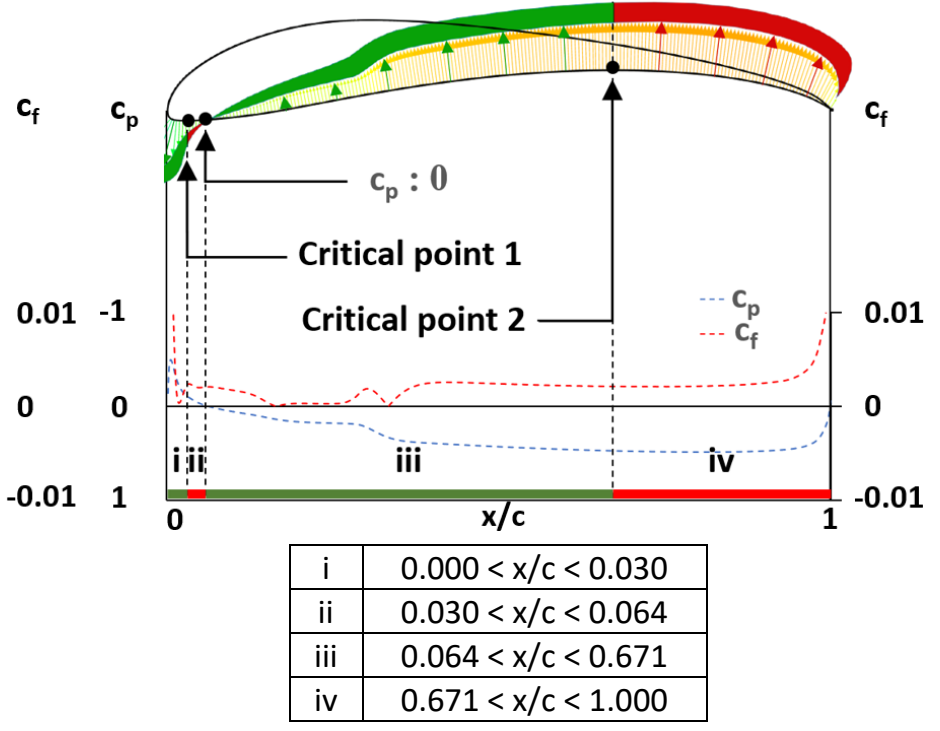

(d) S1223 at $\alpha: 0.46^{\circ}$, Re: $0.3 \times 10^{6}$

Fig. 13. Pressure vectors of the lower surface along with $c_{p}$ and $c_{f}$ distribution as a function of chord length. Green portion represents portion of the airfoil which contributes towards negative pressure drag and red portion represents positive pressure drag

In the context of pressure forces, the airfoils differ most visibly on the onset of positive $c_{p}$ which makes it apparent as to why concave trailing edge airfoils are successful in delivering greater lift. The NACA 4415 and the "partially concave" FX 61-184 have their $c_{p}$ inflection point to be located at around $48.2 \%$ and $59.5 \%$ of chord respectively whereas the S1223 and E420 being significantly further upstream at $6.4 \%$ and $11 \%$, therefore, adding a substantial amount of total lift. Moreover, the considerable deceleration of flow due to the concavity on the lower surface promotes a higher increase in $c_{p}$ with a maximum value of 0.48 for the S1223 and 0.41 for the E420. In relation to transition, the concave airfoils benefited from this as the static pressure slightly increases in the presence of turbulent flow. This can be clearly seen in Figure 13 (d) where a jump in the pressure vectors in region "iii" is visible.

The breakdown of pressure drag on the lower surface of concave airfoils is interesting mainly due to the presence of a second critical point. The first critical point will mark the entry of the flow into 
the concave portion of the airfoil hence undergo deceleration towards a point of minima. The pressure gradient then builds up due to the change in surface curvature gradient and therefore accelerates throughout the remaining length of the airfoil. This results in an additional region aft of the second critical point which contributes to positive pressure drag. The FX 61-184 that can somehow be considered as "partially concave" airfoil has its second critical point to be rather close to the trailing edge at $92.2 \%$ of the chord while "fully concave" airfoils, the E420 and S1223 at 56.4\% and $67.1 \%$ respectively. Despite that, the region denoted as "iii" as shown in Figure 13 (c) and (d) is bounded by the pressure inflection point and the second critical point accommodates the increase by providing some degree of offset. In relation to convex airfoils, the pressure drag composition is made up of 4 regions. Since the NACA 4415 features essentially an almost flat lower surface, the production of pressure forces is typically small thus suggesting that most of the pressure drag is produced by the upper surface and in the vicinity of the stagnation point as shown in Figure 12 (b). The same concept holds true for the NACA 0012.

Figure 14 displays the dependence of the critical point towards the angle of attack at $0^{\circ}$ and the corresponding maximum I/d of the airfoils. The airfoils on the right-hand side are rotated according to the actual angle of attack to showcase the shift of the critical points thus equivalent chordwise stations are slightly distorted. The general takeaway that can be deduced is that the critical points shift with increasing angle of attack and eventually disappear depending on the curvature of the airfoil. This will result in a change in the pressure gradient and is confined to the movement of the critical point. The variation in pressure gradient should also trigger different transition onset locations. In Figure 14 (a) at a $6^{\circ}$ angle of attack, the lower surface of the NACA 4415 has a complete contribution towards pressure drag. This is due to the airfoil being flat bottomed which arrests any generation of strong pressure gradient with increasing angle of attack. Consequently, the flow is only able to accelerate to a very small length downstream upon arriving at a fairly constant velocity. Another noticeable difference on the NACA 4415 is the extent to which the critical point being shifted upstream on the top surface that promoted the onset of transition to occur earlier. In comparison to the E420 and S1223, the curvature gradient is relatively small therefore resulting in a greater upstream shift of the critical point with respect to the angle of attack. Not only this has lengthened the region of positive pressure drag but also presents additional viscous shear to increase the total drag.
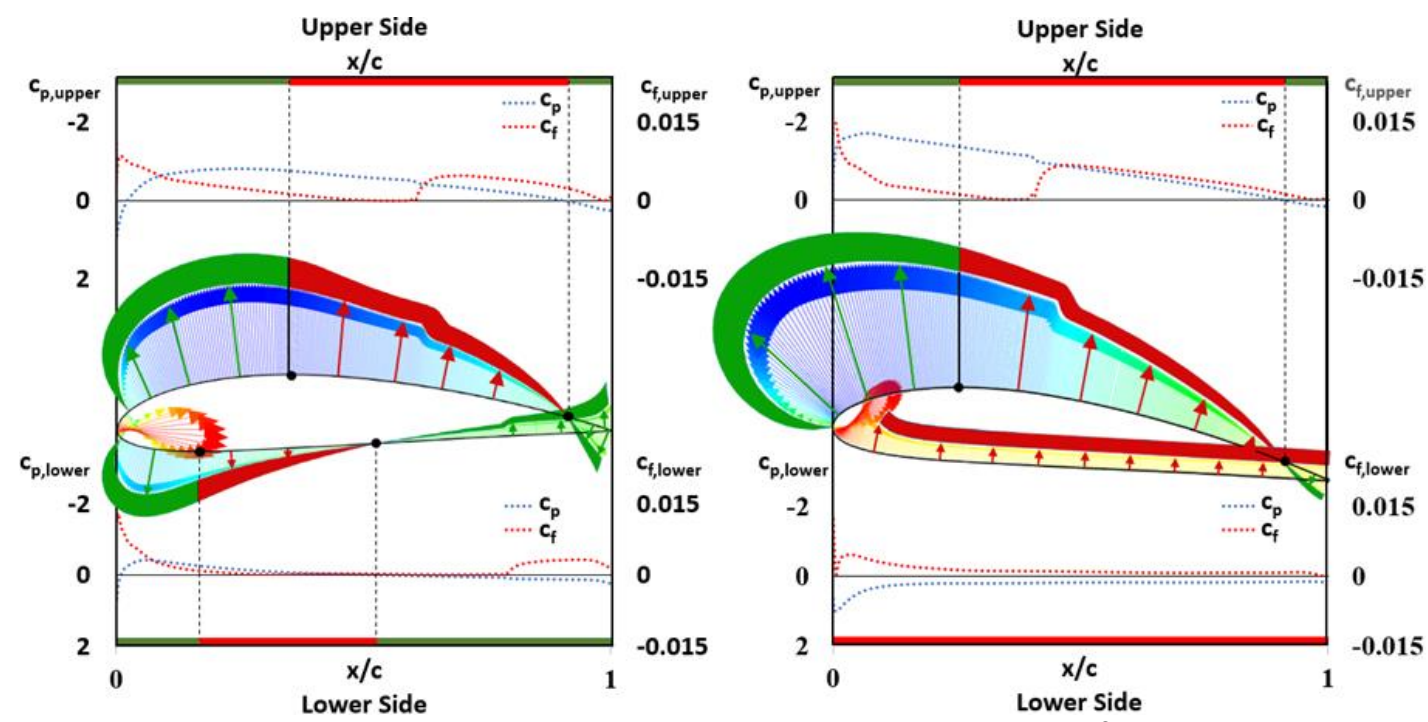

(a) NACA 4415. Left $\left(\alpha: 0^{\circ}\right)$, Right $\left(\alpha: 6^{\circ}\right)$ at Re: $1.0 \times 10^{6}$ 

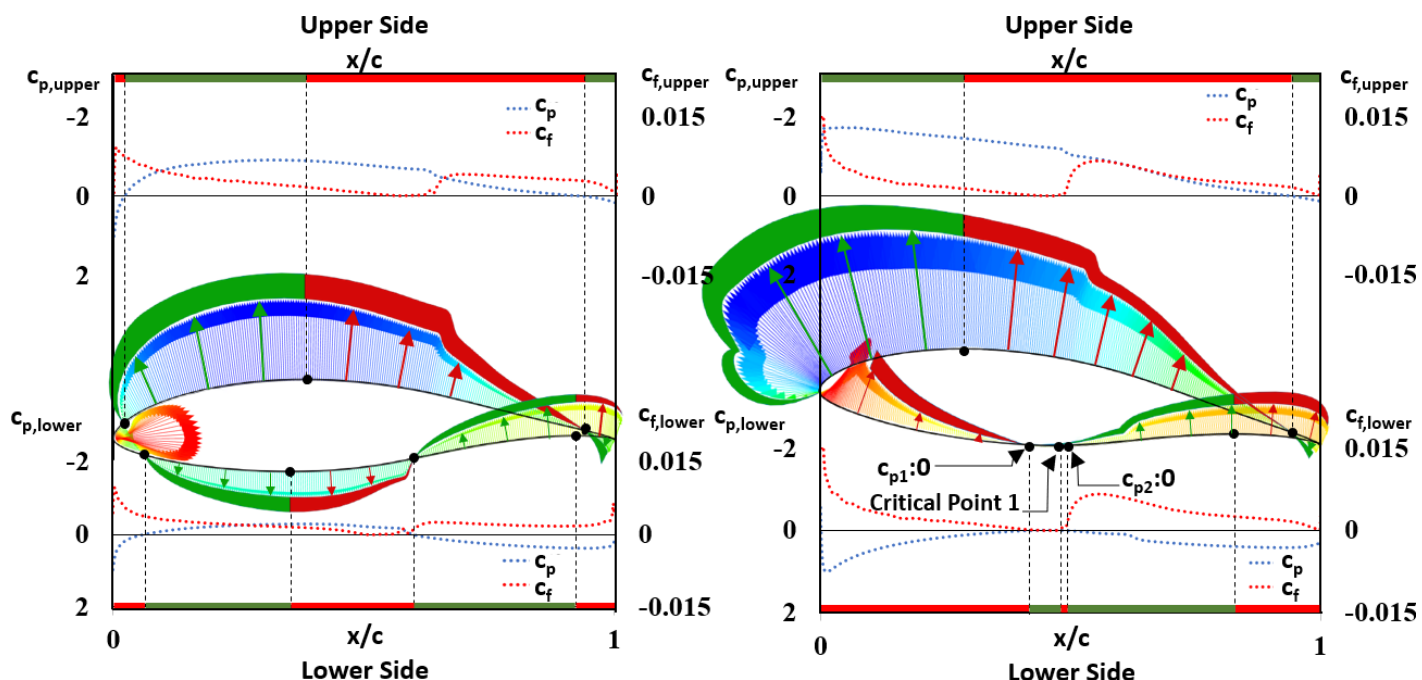

(b) FX 61-184. Left $\left(\alpha: 0^{\circ}\right)$, Right $\left(\alpha: 6^{\circ}\right)$ at Re: $1.0 \times 10^{6}$
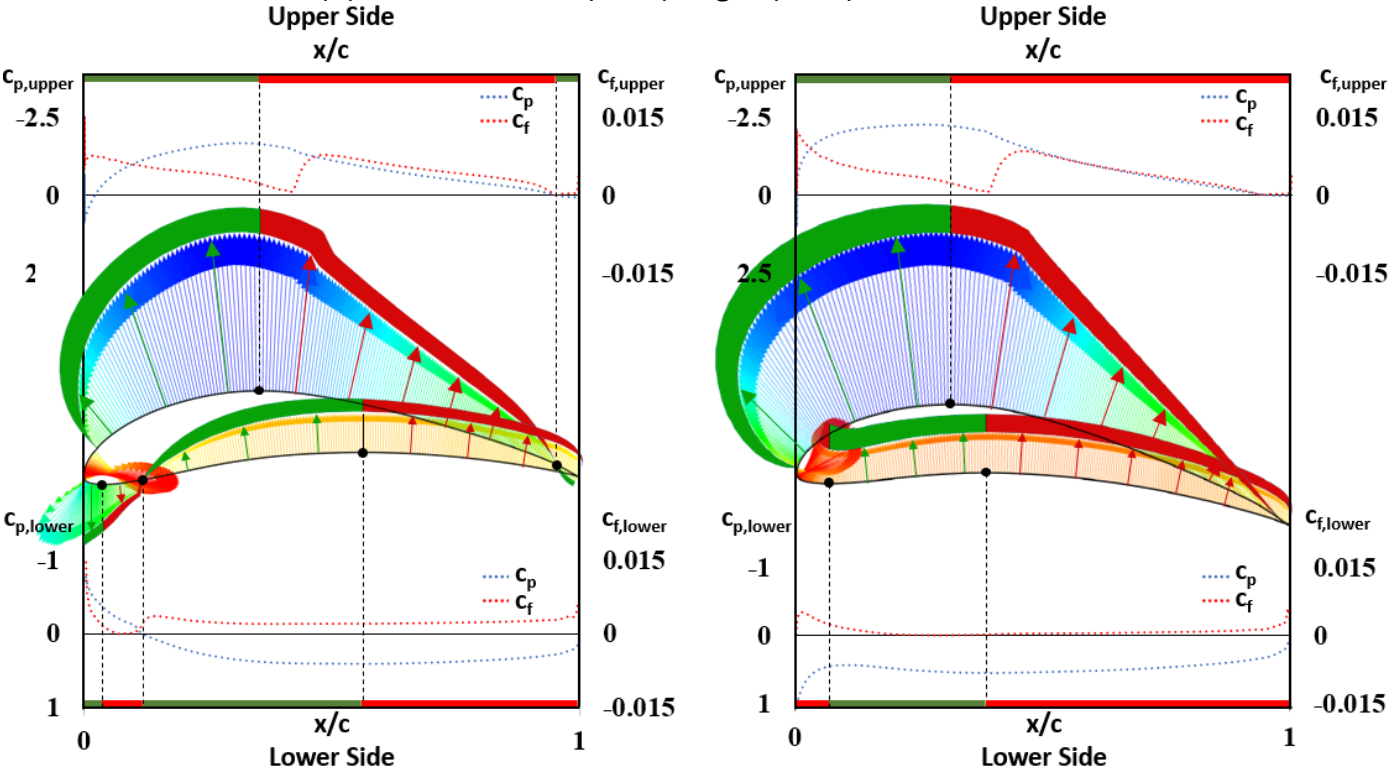

(c) E420. Left $\left(\alpha: 0^{\circ}\right)$, Right ( $\left.\alpha: 6^{\circ}\right)$ at Re: $1.0 \times 10^{6}$
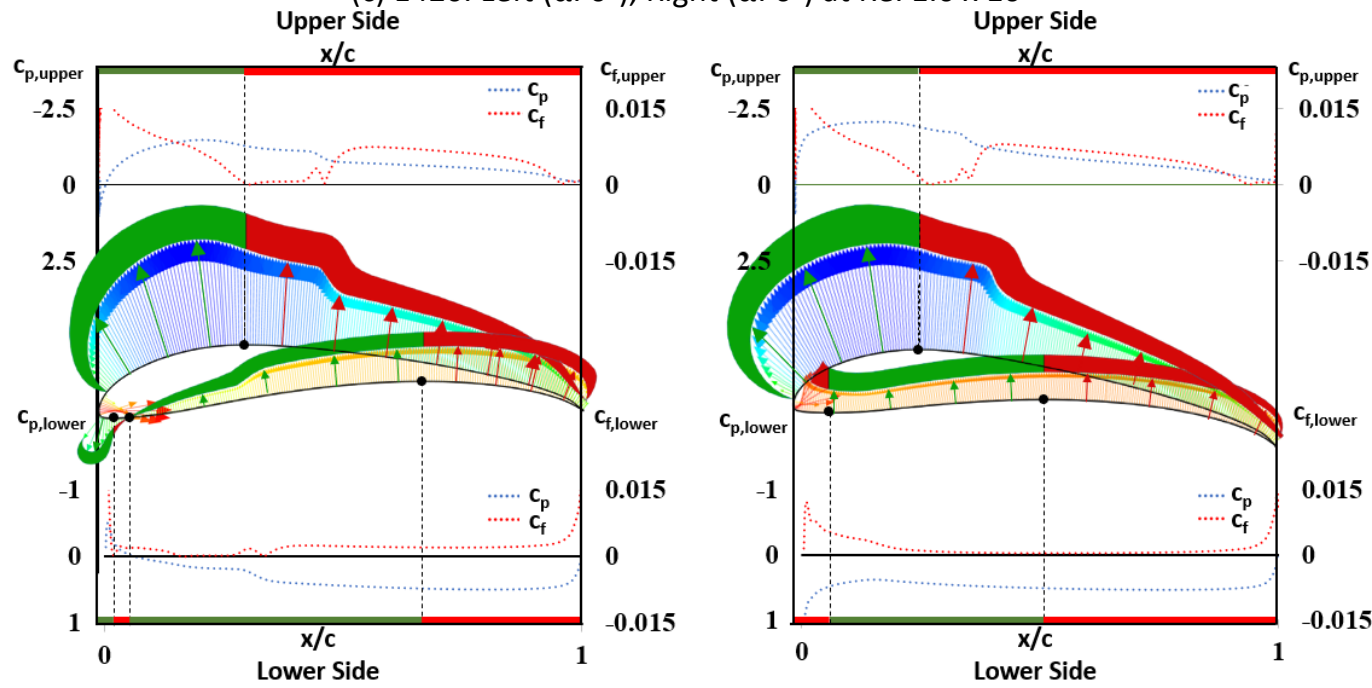

(d) S1223. Left $\left(\alpha: 0.46^{\circ}\right)$, Right ( $\left.\alpha: 5.07^{\circ}\right)$ at Re: $0.3 \times 10^{6}$

Fig. 14. Pressure vectors at the angle of attack of zero and $l / d(\max )$ along with $c_{p}$ and $c_{f}$ distribution as a function of chord length. Green portion represents portion of the airfoil which contributes towards negative pressure drag and red portion represents positive pressure drag. 
On the other hand, in Figure 14 (b) on the right, the convex portion on the lower surface of the FX 61-184 aids the flow to accelerate to about $48 \%$ of the chord before decelerating towards the second critical point. Observe also that the surface curvature near the first critical point is very weak. This induces two pressure inflection points to occur very close to each other, one before the critical point during flow acceleration and another right after the flow decelerates. The contribution of this small portion of negative pressure drag is certainly negligible. Another interesting point to note is that the critical point on this airfoil has actually shifted downstream, as it was at around $35 \%$ initially at $0^{\circ}$ angle of attack. Nevertheless, the change in the critical point to move aft does not accommodate extension of the laminar zone. Looking closely on the lower surface of the FX 61-184 airfoil from the first to the second critical point at an angle of attack of $6^{\circ}$, the variation in curvature does not exactly fall short when compared to that of a straight horizontal line. This thereby dismisses any strong contribution towards neither pressure nor negative pressure drag. In other words, the $40 \%$ of the portion on the lower surface of the airfoil which by definition produces negative pressure drag, in essence gives only a small amount of influence. Needless to say, the greatest offset of the strong pressure drag relies on the negative drag contribution on the top surface.

Concave airfoils generally have a comparable surface curvature on both surfaces. Unlike the previous two airfoils, the shift of the critical point upstream is not as severe. Despite that, a common feature can be extracted where the shift of the critical point upstream will elongate the positive pressure drag after the point of maxima from which the E420 and S1223 will suffer quite seriously. The transition ramp of the S1223 however serves its purpose well at this angle of attack as the separation bubble grows shorter as seen in Figure 14 (d). The lower surface contribution on both airfoils however is similar to that of the FX 61-184 where the negative pressure drag region is a major contributor to lift. On top of that, as the airfoil curvature aft of the second critical point is quite high near the trailing edge, local positive static pressure will surely increase the total drag.

\section{Conclusion}

The breakdown of pressure drag on the NACA 4415, FX 61-184, E420, and S1223 airfoils suitable for UAV application has been studied in this paper. The numerical investigation has displayed that unsteady simulation using the 3-equation Intermittency SST model is capable of predicting transition with relatively good accuracy when compared to XFOIL. Experimental lift and drag validation across all the airfoils generally suggest that the transitional model regularly outperforms XFOIL especially in the case of the $\mathrm{S} 1223$ at a Reynolds Number of $0.3 \times 10^{6}$.

Up to this point, the breakdown of pressure drag has been conceptually understood. Geometrically, the location of the critical and inflection pressure points define different regions on the airfoil which contribute either towards positive or negative drag. Among the selection of airfoils in this study, concave airfoils such as the E420 and S1223 excel in delivering high lift at the expense of an increase in drag which may underestimate its potential. These airfoils should be quite preferable to be integrated into the design of small/medium-sized UAVs due to their high-lift properties. Therefore, further studies should focus on the implementation of drag reduction techniques on concave airfoils to increase their efficiency.

\section{Acknowledgement}

This research was not funded by any grant. 


\section{References}

[1] Eisenbeiss, Henri. "A mini unmanned aerial vehicle (UAV): system overview and image acquisition." International Archives of Photogrammetry. Remote Sensing and Spatial Information Sciences 36, no. 5/W1 (2004): 1-7.

[2] Shakhatreh, Hazim, Ahmad H. Sawalmeh, Ala Al-Fuqaha, Zuochao Dou, Eyad Almaita, Issa Khalil, Noor Shamsiah Othman, Abdallah Khreishah, and Mohsen Guizani. "Unmanned aerial vehicles (UAVs): A survey on civil applications and key research challenges." leee Access 7 (2019): 48572-48634.

https://doi.org/10.1109/ACCESS.2019.2909530

[3] Marqués, Pascual, and Andrea Da Ronch. Advanced UAV Aerodynamics, Flight Stability and Control. Wiley, 2017. https://doi.org/10.1002/9781118928691

[4] Nagel, Alexander, and Misha Shepshelovich. "The concept of high-lift, mild stall wing." In 24th International Congress of the Aeronautical Sciences, Yohogama, Japan, 29August-03 September. 2004.

[5] Selig, Michael S., and James J. Guglielmo. "High-lift low Reynolds number airfoil design." Journal of aircraft 34, no. 1 (1997): 72-79. https://doi.org/10.2514/2.2137

[6] Basri, Adi Azriff, Ernnie Illyani Basri, Mohd Firdaus Abas, Faizal Mustapha, Mohamed Thariq Hameed Sultan, and Kamarul Arifin Ahmad. "UAV NACA4415 wing structural performance analysis subjected to external aerodynamic load using Schrenk's approximation." Journal of Advanced Research in Fluid Mechanics and Thermal Sciences 60, no. 2 (2019): 178-190.

[7] Althaus, Dieter. Experimental results from the Laminar Wind Tunnel of the Institut für Aero-und Gasdynamik der Universität Stuttgart. Vol. 1. F. Vieweg, 1981.

[8] Eppler, Richard. Airfoil design and data. Springer Science \& Business Media, 2012.

[9] Windte, Jan, Rolf Radespiel, Ulrich Scholz, and Bernhard Eisfeld. RANS simulation of the transitional flow around airfoils at low Reynolds Numbers for steady and unsteady onset conditions. Technical Univ Braunschweig (Germany) Inst Of Fluid Mechanics, 2004.

[10] Langtry, Robin Blair, F. R. Menter, S. R. Likki, Y. B. Suzen, P. G. Huang, and S. Völker. "A correlation-based transition model using local variables - part II: test cases and industrial applications." (2006): 423-434.

https://doi.org/10.1115/1.2184353

[11] Khayatzadeh, Peyman, and Siva Nadarajah. "Laminar-turbulent flow simulation for wind turbine profiles using the p-transition model." Wind Energy 17, no. 6 (2014): 901-918. https://doi.org/10.1002/we.1606

[12] Lanzafame, R., S. Mauro, and M. Messina. "Wind turbine CFD modeling using a correlation-based transitional model." Renewable Energy 52 (2013): 31-39.

https://doi.org/10.1016/i.renene.2012.10.007

[13] Inc ANSYS, ANSYS Fluent Theory Guide (release 2020 R2). 2020, Ansys Canonsburg, PA.

[14] Menter, Florian R., Robin Blair Langtry, S. R. Likki, Y. B. Suzen, P. G. Huang, and S. Völker. "A correlation-based transition model using local variables-part I: model formulation." (2006): 413-422. https://doi.org/10.1115/1.2184352

[15] Langtry, Robin Blair. "A correlation-based transition model using local variables for unstructured parallelized CFD codes." (2006).

[16] Keating, Mark, and A. N. S. Y. S. Principal Engineer. "Accelerating CFD solutions." advantage 1 (2011): 48.

[17] Drela, Mark. "XFOIL: An analysis and design system for low Reynolds number airfoils." In Low Reynolds number aerodynamics, Springer, (1989): 1-12. https://doi.org/10.1007/978-3-642-84010-4 1

[18] Selig, Michael S. Summary of low speed airfoil data. SOARTECH publications, 1995.

[19] Sheldahl, R. E., and P. C. Klimas. "Aerodynamic characteristics of seven airfoil sections through 180 degrees of attack for use in aerodynamic analysis of vertical axis wind turbines, SAND80-2114." Sandia National Laboratories, Albuquerque, NM (1981). https://doi.org/10.2172/6548367

[20] Miley, Stan J. Catalog of low-Reynolds-number airfoil data for wind-turbine applications. No. RFP-3387. Rockwell International Corp., Golden, CO (USA). Rocky Flats Plant; Texas A and M Univ., College Station (USA). Dept. of Aerospace Engineering, 1982.

https://doi.org/10.2172/5044823

[21] Wortmann, F. X. "A critical review of the physical aspects of airfoil design at low Mach numbers." NASA CR 2315 (1973): 179-196.

[22] Mangalam, S., J. R. Dagenhart, J. Meyers, and T. Hepner. "The Görtler instability on an airfoil." In 23rd Aerospace Sciences Meeting, p. 491. 1985.

https://doi.org/10.2514/6.1985-491 